5

8 2Department of Immunology and Microbial Disease, Albany Medical College, Albany, NY

912208

$10{ }^{3}$ National Institute on Aging-National Institutes of Health, 08C218,Biomedical Research Center,

14

15

16

17

18

19

20

\section{TCF-1 in CD4 T cells regulates GVHD severity and persistence} 251 Bayview Boulevard, Suite 100, Baltimore, MD 21224

${ }^{4}$ Immunology Program, Department of Medicine, Johns Hopkins School of Medicine, Baltimore, MD 21224.

To whom correspondence should be addressed:

Mobin Karimi

Assistant Professor of Immunology and Microbiology

SUNY Upstate Medical University,

766 Irving Ave Weiskotten Hall Suite 2281,

Syracuse, NY 13210

Office Phone: 315-464-2344

Laboratory Phone: 315-464-7652

Email: karimim@upstate.edu

Conflict of interest statement: The authors have declared that no conflict of interest exists. 


\section{Abstract}

37 Graft-versus-host disease (GVHD) is a leading cause of mortality following allogeneic

38 hematopoietic stem cell transplantation (allo-HSCT). Mature donor T cells in the graft mediate graft-versus-leukemia (GVL) responses against residual tumor cells, which may persist after pretransplant conditioning regimens. Importantly, the same mature T cells also mediate GVHD. The transcription factor T Cell Factor-1 (TCF-1) is critical for T cell development in the thymus. Using a unique mouse model of allo-HSCT leading to GVHD, we investigated the role of TCF-1 in alloactivated T cell functioning and in GVHD. Here, we report that loss of TCF-1 in mature CD4 T cells reduces GVHD severity and persistence, improving survival of recipient mice. This was due to reduced proliferation, survival, and cytokine production of T cells, as well as increased exhaustion. Gene pathways involved in cytokine response, immune signaling,

47 chemokine signaling, cell cycle, and T cell differentiation were altered by loss of TCF-1 in donor cells. Our companion paper shows that regulation of alloactivated CD4 T cells by TCF-1 differs

49 from regulation of CD8 T cells, suggesting that TCF-1 plays a unique role in each subset.

50 Therefore, targeting of TCF-1 or downstream signaling pathways may be an effective strategy

51 for reducing GVHD following allo-HSCT. 
62

63

64

65

66

67

68

69

70

71

72

73

74

75

76

77

78

79

80

81

82

83

84

85

86

\section{Introduction}

Patients with hematological malignancies often undergo allogeneic hematopoietic stem cell transplantation (allo-HSCT), a curative therapy (1). This involves donor stem cells being transplanted, along with mature donor T cells (2). These mature T cells in the graft help to clear residual malignant cells and prevent relapse, an outcome called the graft-versus-leukemia (GVL) effect. However, 30-70\% of these patients also develop graft-versus-host disease (GVHD), a lifethreatening complication mediated by these same donor T cells (3). Alloactivation of the T cells by non-self HLA leads to T cell proliferation, migration, and production of cytokines, resulting in damage to healthy tissues (4). GVHD remains a major clinical barrier to widespread use of allo-HSCT, and depletion of T cells or immunosuppressive therapies are sub-optimal, leaving the patient susceptible to infection or relapse (5). Our research seeks ways to modulate mature T cell signaling during alloactivation to separate the linked processes of GVHD and GVL. To reach this goal, better understanding is needed on how alloactivated T cells are regulated. T Cell Factor-1 (TCF-1) is a T-cell transcription factor which is critical for T cell development $(6,7)$. TCF-1 is also known to be involved in T cell proliferation, recall, and memory responses during viral infection (8). However, to our knowledge, TCF-1 has not been studied in the context of alloactivation, which is a different process from canonical activation resulting from recognition of non-self HLA rather than of pathogens (9). TCF-1 has also been reported to affect the transcription factor Eomesodermin (Eomes) and possibly T-box transcription factor 21 (T-bet) $(10,11)$. Eomes and T-bet affect both GVHD and GVL, but it remains unknown whether TCF-1 may play a role upstream of these factors in separating GVHD from GVL (12-15) . 
87 Global deletion of TCF-1 significantly reduces the number of thymocytes, as well as the number

88 of mature T cells exiting the thymus, because TCF-1 is critical for the double-negative stages of

89 development (16-18). To overcome this problem, we obtained mice that have a T cell-specific

90 deletion of TCF-1 using CD4cre (Tcf7 flox/flox x CD4cre) $(19,20)$. These mice are able to

91 produce mature T cells because they retain TCF-1 expression until the double-positive phase of

92 development in the thymus(20). At this point, all T cells will have TCF-1 expression silenced by

93 CD4cre (because all T cells express CD4 at this point, even CD8 T cells)(20). Therefore, this

94 unique mouse strain allows us to study mature T cells with TCF-1 deficiency.

95 Using a mouse model of GVHD and GVL following allo-HSCT, we found that loss of

96 TCF-1 in CD4 T cells reduces both the severity and the persistence of GVHD, leading to

97 improved survival of recipient mice following transplant. This disease reduction was driven by

98 alterations to $\mathrm{T}$ cell proliferation, cytokine production, survival, and exhaustion, as well as

99 changes in gene expression. Expression of chemokine receptors on donor T cells was enhanced

100 by loss of TCF-1. We also showed that loss of TCF-1 in CD4 T cells induces T cell-intrinsic

101 phenotypic changes to memory identity, including increased CD4 naive cells and decreased

102 activating/transitioning CD4 T cells. TCF-1 also affects expression of genes in the cytokine

103 response, cell cycle control, T cell differentiation, and immune signaling pathways, among

104 others. Therefore, TCF-1 controls mature CD4 T cell phenotype, functions, and gene expression 105 during alloactivation, a novel finding.

106 Here, we provide evidence that TCF-1 regulates mature alloactivated T cell function and 107 genetic programs, thereby controlling GVHD severity and persistence. The major alloactivated T 108 cell functions - cytokine production, migration, and proliferation - were altered by loss of TCF109 1, as were cell survival and gene expression. Our companion manuscript shows that the control 
110 of CD4 T cells by TCF-1 is distinct from control of CD8 T cells. Therefore, TCF-1 acts as a

111 regulatory transcription factor in mature alloactivated CD4 T cells, and modulation of TCF-1 and

112 downstream signaling may be a successful option for reducing GVHD to improve patient

113 outcomes following allo-HSCT.

114

115

116

117

118

119

120

121

122

123

124

125

126

127

128

129

130

131

132

133

134

135

136

137

138

139

140

141

142

143 


\section{Results}

Loss of TCF-1 in donor CD4 T cells reduces severity and persistence over time of GVHD

147 symptoms. Previous research on TCF-1 has focused on T cell development and canonical

148 activation $(21,22)$. These studies primarily used a global TCF-1 knockout, resulting in limited

149 production of mature T cells $(21,22)$. We sought to examine the role of TCF-1 in mature T cells,

150 so to overcome this limitation we employed mice with T-cell-specific deletion of TCF-1 using

151 CD4cre (20). This mouse strain has TCF-1 deleted in all CD4 and CD8 T cells at the DP phase,

152 allowing production of mature T cells with TCF-1 deletion. To investigate whether TCF-1 in

153 mature CD4 T cells contributes to GVHD after allo-HSCT, we employed a murine model of

154 MHC-mismatched allotransplantation. Donor CD4 T cells from C57Bl/6 background wild-type

155 (WT), CD4cre control, or TCF-1-deficient (TCF cKO) mice were injected along with $\mathrm{T}$ cell

156 depleted BALB/c bone marrow into lethally irradiated BALB/c recipients as described in the

157 methods $(15,23)$. The MHC haplotype mismatch ( $\mathrm{H} 2 \mathrm{~Kb}$ in donors, $\mathrm{H} 2 \mathrm{Kd}$ in recipients) results

158 in alloactivation of the donor T cells, leading to GVHD (15). Recipient mice were then weighed

159 and given a GVHD score (Fig.1A) to identify the severity of GVHD for up to 60 days post-

160 transplant. Recipient mice were scored based on weight loss, fur texture, posture, activity, skin

161 condition, and diarrhea, as previously described(15, 24).

162 Mice receiving CD4 T cells from WT or CD4cre donors experienced a rapid increase in

163 GVHD symptoms, peaking at a very high score, indicating very severe disease (25). CD4 T cells

164 are known to cause very severe GVHD symptoms, so this finding was expected (25). Over time,

165 these recipient mice continued to show severe symptoms, with consistent scores until death from

166 disease (Fig.1A). Survival was also poor, with most mice in this group dying prior to day 10

167 (Fig.1B). These mice lost weight due to disease, and died before they were able to regain much 
168 weight (Supp. Fig.1A). The disease burden, as determined by total number of mice per group

169 that died by the end of the experiment, was very high for WT and CD4cre-transplanted mice

170 (Fig.1C). In contrast, mice receiving CD4 T cells from TCF cKO mice showed reduced GVHD

171 scores (Fig.1A), better survival (Fig.1B), and weight gain following initial weight loss (Supp.

172 Fig.1A). Disease burden was reduced in this group (Fig.1C), with most mice in this group

173 remaining alive at day 60 post-transplant. Interestingly, mice in the TCF cKO donor group still

174 showed a peak in GVHD score early on, as with the WT group, but this peak was at a much

175 lower score. Additionally, this peak score did not persist over time, as the scores for these mice

176 quickly reduced to the level seen in bone marrow-only controls (Fig.1A). In addition, this low

177 score remained low for an extended period of time, suggesting that disease had resolved rather

178 than been delayed (Fig.1A). These data indicated that GVHD symptoms were not only less

179 severe in these mice, but also less persistent over time.

180 To examine pathological damage to target organs - including the liver, guts, and skin -

181 tissues from these organs were collected for histology at three time points. At day 7, day 14, and

182 day 21 post-transplant, organs were taken for histology, H\&E stained, and scored for GVHD

183 pathology. At day 7, little to no difference was observed in tissue damage or GVHD grade

184 between WT and TCF cKO transplanted mice (Fig.1D-E), (Supp.Fig.1B), in agreement with the

185 GVHD score data, where major differences occurred after day 7. By day 21, differences in

186 GVHD grade were observed between WT and TCF cKO recipients, with lymphocyte infiltration

187 visible in liver, guts, and skin of WT but not TCF cCKO-transplanted mice (Fig.1D-E), (Supp.

188 Fig.1C). In addition, tissue damage in guts and skin are clearly visible for WT but not TCF cKO-

189 transplanted mice (Fig.1D). Splenic structural disorganization is also visible in spleen of WT but

190 not TCF cKO-transplanted mice (Supp.Fig.1C). By day 14, these changes are already visible as 
191 a trend in GVHD grade, indicating a progressive resolution of GVHD symptoms over time in

192 TCF cKO-transplanted mice (Supp. Fig.1B). By day 21, the percentage of mice experiencing

193 severe GVHD (grades 3-4, versus no or mild to moderate GVHD at grades 0-2.(15, 26)(27) was

194 trending towards increased for WT-transplanted mice, compared to TCF cKO-transplanted mice,

195 and this trend can be seen as early as day 14 (Fig.1E). At day 7, there is no apparent difference

196 between the distribution of disease grades (Fig.1E). For all organs, the histology grade tends to

197 decrease over time for TCF cKO-transplanted mice, but rise or remain consistent for WT-

198 transplanted mice (Supp. Fig.1B), further supporting the idea that disease resolves over time and

199 does not persist when donor cells are TCF-1-deficient. Together, these results indicate that TCF-

2001 normally contributes to and is indispensable for GVHD damage by T cells, and loss of TCF-1

201 reduced severity and persistence of GVHD.

202

203

204

205

206

207

208

209

210

211

212

213

Loss of TCF-1 drives changes to mature CD4 T cell memory identity which are primarily cell-

intrinsic. TCF-1-deficiency in CD4 T cells alters GVHD outcomes, suggesting that T cell phenotypes or function are affected. To examine the phenotype of mature CD4 T cells when TCF-1 is lost, we performed flow cytometry phenotyping on naive CD4 T cells from WT and TCF cKO mice (Fig.2A-B), (Supp. Fig.2A-C). We began by looking at expression of the transcription factors Eomesodermin (Eomes) and T-box transcription factor 21 (T-bet), which are downstream of TCF-1. Previous reports suggested that TCF-1 normally activates Eomes, and may activate or not impact T-bet (28). However, we found that loss of TCF-1 in CD4 T cells did not affect expression of Eomes and T-bet (Supp. Fig.2A-B). In addition, expression of CD122 was not impacted by loss of TCF-1 (Supp. Fig.2C). Interestingly, expression of CD44 was polarized more potently in TCF cKO T cells (Fig.2A-B). Using CD62L and CD44, we identified 
214 four major memory subsets: central memory (CDD44+ CD62L+), effector memory (CD44+

215 CD62L-), naive (CD44-), and activating/transitioning (CD44 mid). Comparing WT and TCF

216 cKO mice, we found that loss of TCF-1 results in an increase in CD4+ naive T cells and a

217 decrease in CD4+ transitioning/activating cells compared to WT mice (Fig.2A). These results

218 suggest that loss of TCF-1 results in a more naive phenotype in CD4+ T cells. Given the

219 increased ability of naive cells to cause severe GVHD, this would suggest that loss of TCF-1

220 would increase GVHD severity. Thus, some other T cell functions must be impacted to explain

221 the reduction observed.

222 To investigate whether these changes were cell-extrinsic or -intrinsic, we utilized a

223 chimeric bone marrow mouse model, as described in the methods. Briefly, Thy 1.1 mice were

224 lethally irradiated and reconstituted with bone marrow from WT and TCF cKO donors, mixed at

225 a 4:1 (TCF:WT) ratio, for a total of $50 \times 10^{6}$ BM cells. Due to our initial observations that TCF

226 cKO T cells did not proliferate well in culture, we used a 4:1 ratio to ensure survival of enough

227 TCF cKO bone marrow cells to perform downstream analyses. At 10 weeks post-transplant,

228 splenocytes were obtained from the recipient mice and analyzed by flow cytometry, with donor

229 cells identified by $\mathrm{H} 2 \mathrm{~Kb}$ expression. The increase in naive CD4+ T cells, as well as the reduction

230 in activating cells, was found to be cell-intrinsic in the TCF cKO T cells, because the differences

231 were maintained despite development in a chimeric mouse (Fig.2C). Thus, the memory identity

232 changes induced by loss of TCF-1 are cell-intrinsic. Of note, CD122 expression was increased

233 among TCF cKO donor cells from chimeric mice (Fig.2D), suggesting that these cells are

234 capable of being activated. Again, no differences were seen in Eomes and T-bet expression

235 (Supp. Fig. 2D-E). 

receptor expression in mature CD4 T cells during alloactivation. Our data show that GVHD is disrupted when TCF-1-deficient CD4 T cells are used for allotransplantation. We next sought to determine which of the major alloactivated $\mathrm{T}$ cell functions - migration, proliferation, and cytokine production - were altered to provide the optimal clinical phenotype. First, we examined CD4 T cell migration by transplanting lethally irradiated BALB/c mice with WT and TCF cKO total $\mathrm{CD} 3+\mathrm{T}$ cells mixed in a 1:1 ratio $\left(1 \mathrm{X} 10^{6}\right.$ total cells), along with $\mathrm{T}$ cell-depleted BALB/c bone marrow (BM, $10 \times 10^{6}$ cells). The donor cells were checked prior to transplant for CD45.1/CD45.2 (WT and TCF cKO congenic markers, respectively) (Fig.3A), (Supp. Fig.3). CD4 and CD8 T cells were first mixed 1:1 for each strain, then a 1:1 mixture of the two strains was prepared. WT CD45.1 and WT CD45.2 cells were mixed in the same way as a control. After 7 days, the spleen, lymph nodes, small intestine (SI or "gut"), and liver were removed from recipients and lymphocytes were obtained (15). frequency of TCF cKO CD4 T cells would be expected to be higher than WT in the spleen and reduced survival or a potential defect in proliferation. 
260

261

262

263

264

265

266

267

268

269

270

271

272

273

274

275

276

277

278

279

280

281

282

donor cells) and performed qPCR using a 96-well mouse chemokine/chemokine receptor array plate (ThermoFisher). We found that the expression of chemokines and chemokine receptors was upregulated following alloactivation, as expected. However, expression of these markers was consistently higher in TCF cKO CD4 T cells from spleen, both pre- and post-transplant (Fig.3BC), while these markers were downregulated in TCF cKO CD4 T cells from post-transplant liver (Fig.3D). Therefore, CD4 T cells from TCF cKO mice should be capable of migration at normal levels, and the observed difference in migration is likely due to changes in proliferation or cell survival. However, it is possible that these cells may have a mild migration defect, which could become more apparent later in the disease course.

TCF-1 controls proliferation, apoptosis, and exhaustion of mature alloactivated CD4 T cells.

The donor T cells responsible for GVHD must proliferate in the secondary lymphoid organs and in the GVHD target organs in order for the response to continue (29). Effector T cells - which drive GVHD - have a short lifespan, so they must be continually replaced during an alloresponse. To investigate whether loss of TCF-1 affects the proliferation of mature alloactivated $\mathrm{T}$ cells, we transplanted lethally irradiated BALB/c mice with $1 \mathrm{X} 10^{6} \mathrm{WT}$ or TCF cKO CD3 T cells and $10 \times 10^{6} \mathrm{BALB} / \mathrm{c} \mathrm{BM}$, as described above. The recipient mice were injected with EdU in PBS at $25 \mathrm{mg} / \mathrm{kg}$ on day 5-6. At day 7, recipient mice were euthanized, lymphocytes were obtained from the spleen and liver, and donor cells (identified by $\mathrm{H} 2 \mathrm{~Kb}, \mathrm{CD} 3$, and $\mathrm{CD} 4$ ) were tested for the presence of EdU with a click chemistry flow cytometry kit. A trend of increased proliferation in the spleen and significantly increased proliferation in the liver by TCF cKO donor CD4 T cells was observed (Fig.4A-B). Therefore, proliferation at day 7 is increased in TCF cKO CD4 T cells. We also used expression of Ki67 in a similar experiment to examine the activation or 
283 proliferation of these donor cells, and found that activation/proliferation of $\mathrm{T}$ cells was trending

284 towards increased for TCF donor cKO CD4 T cells in the spleen, but not in the liver (Fig.4C-D).

285 Therefore, proliferation in T cells early after alloactivation is increased by loss of TCF-1.

286 However, given that symptoms of GVHD resolve after day 7 in TCF cKO-transplanted

287 recipients, we hypothesize that proliferation of donor cells also decreases below WT levels after 288 this point.

To examine the role of TCF-1 in survival of mature T cells during alloactivation, we

290

291

292

performed a death assay using flow cytometry. Recipient mice were allotransplanted in the same manner as for proliferation experiments, and at day 7, cells from the spleen and liver were analyzed by flow cytometry. Donor cells were detected with $\mathrm{H} 2 \mathrm{~Kb}, \mathrm{CD} 3, \mathrm{CD} 4$, and CD8. Using Annexin V-FITC and LIVE/DEAD Near IR, we were able to separate three distinct populations live cells, dead cells, and apoptotic cells (Fig.4E-F). When we compared WT and TCF cKO donor cells, we found that TCF cKO CD4 T cells were trending towards more frequently dead, and less frequently live or apoptotic, than WT donor cells (Fig.4E-F). In support of this, when we attempted to sort CD4 T cells from WT- and TCF cKO-transplanted mice at day 7, there were significantly fewer CD4 donor T cells in the spleen from TCF cKO donors than from WT donors (by number of cells/10,000 total cells) (Supp. Fig. 4A-B). In addition, we attempted to sort TCF cKO donor cells from recipient mice at day 14 post-transplant, and donor cells in the spleen were significantly reduced (with cells in liver trending to reduced) compared to WT and TCF cKO donor T cells from day 7 (Supp. Fig. 4A-B). Empirical observations from our work also suggested that TCF cKO T cells proliferate poorly in culture compared to WT T cells, even when activated. Therefore, it is likely that survival and/or proliferation are reduced further after day 7 , giving rise to the progressive resolution in symptoms observed in TCF cKO-transplanted mice. 
Finally, to determine whether exhaustion of the T cells occurred when TCF-1 was lost,

307

308

309

310

311

312 313 cells.

314

315

316

317

318

319

320

321

322

323

324

325

326

327

we allotransplanted mice as described above, and assessed donor cells from the spleen and liver

at day 7 post-transplant for the expression of TOX, a T-cell exhaustion marker. We found that donor CD4 T cells from TCF cKO mice were significantly more exhausted in the liver, and

trending towards more exhausted in the spleen, than WT donor cells (Fig.4G-H). Together, these data suggest that CD4 donor T cells from TCF cKO mice have worse survival, increased exhaustion, and a potential progressive defect in proliferation over time compared to WT CD4 T

\section{Loss of TCF-1 changes the cytokine profile of mature alloactivated CD4 T cells.}

Production of inflammatory cytokines, eventually culminating in a cytokine storm, in considered a hallmark of GVHD $(29,30)$. Cytokines and cytotoxic mediators are essential for T cells to maintain the GVL effect and kill tumor cells, yet they also lead to damage of healthy host tissues $(30,31)$. To examine cytokine production by TCF cKO CD4 T cells, we allotransplanted recipient mice as described above with $1.5 \times 10^{6} \mathrm{CD} 3$ donor T cells (WT or TCF cKO) and 10X10 $10^{6} \mathrm{BALB} / \mathrm{c} \mathrm{BM}$. At day 7 post-transplant, splenocytes were obtained from recipient mice, and were restimulated to induce cytokine production. Cells were restimulated with Golgiplug along with PBS (negative control) or anti-CD3/anti-CD28 (restimulated) for 6 hours at 37C. After this incubation, the cells were stained for flow cytometry with antibodies against $\mathrm{H} 2 \mathrm{~Kb}$, CD3, CD4, CD8, TNF- $\alpha$, and IFN- $\gamma$. We found that production of TNF- $\alpha$ by donor cells trended toward decreasing when TCF-1 was lost (Fig.5A). On the other hand, production of IFN- $\gamma$ did not appear to be affected by loss of TCF-1 (Fig.5B). 
We also took blood from these recipient mice and obtained serum, which we tested for

329

330

331

332

333

334

335

336

337

338

339

340

341

342

343

344

345

346

347

348

349

350

various cytokines using a LEGENDplex ELISA kit (Biolegend). TNF- $\alpha$ was found to be

increased at day 7 in TCF cKO T cells, but then dropped below WT levels by day 14 (Fig. 5C).

By day 14, the trend towards decreased TNF- $\alpha$ in serum of mice transplanted with TCF cKO

donor cells was already visible, suggesting a progressive drop in TNF- $\alpha$ production by donor

cells (Fig.5D). IFN- $\gamma$ was also increased for TCF cKO T cells at day 7 , and dropped to WT

levels by day 14 (Fig.5E). The difference in IFN- $\gamma$ results from serum and donor splenocytes

suggests that loss of TCF-1 in donor cells results in increased production of IFN- $\gamma$ from host

cells early on, compared to in WT-transplanted mice. These data also suggest that TNF- $\alpha$ is more

directly critical for GVHD damage than IFN- $\gamma$ is. Finally, IL-2 expression in serum was

increased at day 7 in TCF cKO-transplanted mice, but then dropped to WT levels by day 14

(Fig.5F). This correlated with the increased proliferation seen at day 7 with EdU, and the

hypothesized drop in proliferation later on. These data suggest that allotransplanted TCF cKO T

cells are more activated early on in the response, but are less active (or less present) later on.

Loss of TCF-1 changes the suppressive profile of mature CD4 T cells. Given that loss of TCF-1

altered mature CD4 T cell phenotype and function, we sought to understand whether suppressive markers on the T cells were also altered. Expression of markers such as CTLA-4, PD-1, and PD-

L1 play a role in how alloactivated T cells interact with other cells. To examine this, we

allotransplanted recipient BALB/c mice with WT or TCF cKO CD4 T cells, and phenotyped donor cells from spleen and liver at day 7, day 14, and day 21 post-transplant. PD-1 expression

was significantly reduced in the spleen and liver for TCF cKO CD4 T cells (Supp. Fig. 5A-B).

This suggests that TCF-1-deficient cells may be less sensitive to suppression by other cells 
351 expressing PD-L1, and therefore may be more activated during disease(29). We also found that

352 expression of PD-L1 was increased in the liver only for TCF cKO CD4 T cells at day 14 (Supp.

353 Fig.5C-D. It is unclear what role PD-L1 may play when expressed on T cells, but it is thought to

354 promote apoptosis of and suppress activation of other nearby T cells (30). This suggests that

355 TCF-deficient CD4 T cells may hinder activation and survival of other T cells. Therefore, loss of

356 TCF-1 promotes a T cell phenotype that is less susceptible to suppression yet potentially more

357 suppressive of other cells. Finally, expression of CTLA-4 was not impacted by loss of TCF-1

358 (Supp. Fig.5E-F). This suggests that TCR signaling activity is not aberrantly regulated by

359 CTLA-4 in TCF-deficient CD4 T cells, so the cells are likely not over- or understimulated

360 through the TCR. Therefore, loss of TCF-1 changes the suppressive profile of mature CD4 T

361 cells, but does not disturb control of TCR signaling by CTLA-4.

362

363

TCF-1 contributes to control of cytokine and immune signaling pathways in donor CD4 T

364

cells. To understand the molecular mechanisms behind the changes we saw in the TCF cKO

donor CD4 T cells, and to understand the role of TCF-1 in regulating gene expression in these

cells, we employed RNA sequencing. We allotransplanted mice with WT or TCF cKO CD3 T

367

cells and BALB/c BM as described above. A FACS-sorted pre-transplant sample of CD4+ donor

368 cells was taken and stored in Trizol. At day 7 post-transplant, donor T cells were sorted back

369 from spleen and liver of recipients using $\mathrm{H} 2 \mathrm{~Kb}, \mathrm{CD} 3, \mathrm{CD} 4$, and CD8. The sorted cells were all

370 stored in Trizol, then sent to the SUNY Upstate Molecular Analysis Core for RNA extraction

371 and library prep (https://www.upstate.edu/research/facilities/molecular-analysis.php). Prepped

372 samples were then sequenced at the University of Buffalo Genomics Core (paired end

373 sequencing on an Illumina NovaSeq 6000, http://ubnextgencore.buffalo.edu) (Fig. 6). After 
374 analyzing the data in Partek Flow, we found that for both pre- and post-transplant samples, WT

375 and TCF cKO donor CD4 T cells clustered separately (Fig. 6A), (Supp. Fig.6A-C). In addition,

376 WT and TCF cKO donor CD4 T cells appeared to be more similar genetically after allo-

377 transplantation, as the number of significantly different genes was much higher in pre-transplant

378 spleen than in post-transplant spleen and liver (Fig. 6B). In pre-transplant spleen, upregulation of

379 Ccr2, Cxcr6, Ccr3 (chemokine receptors) and Mki67 (proliferation) was observed in TCF cKO

380 donor cells, with downregulation of Tox2 (exhaustion) (Fig. 6C). In post-transplant spleen and

381 liver, Ccr9, Ccl5, and Cxcr5 were altered by loss of TCF-1 in donor CD4 T cells (Fig. 6D). This

382 suggests that chemokines and receptors are indeed regulated in mature and alloactivated CD4 T

383 cells by TCF-1, as shown by our qPCR data.

384 Pathway enrichment analysis showed that cell cycle control, T cell differentiation, TCR

385 signaling, FoxO signaling, and immune signaling pathways (such as NOD and RIG-I) were

386 altered by loss of TCF-1 in pre-transplant donor cells (Fig. 6E). Post-transplant,

387 cytokine/cytokine receptor interaction genes were affected in both liver and spleen, and in liver

388 only, other pathways such as intestinal immunity and NOD signaling were also impacted by loss

389 of TCF-1 (Fig. 6F-G). Interestingly, WT and TCF cKO donor CD4 T cells appear to be more

390 different prior to transplant as compared to post-transplant, because the number of pathways

391 disrupted is fewer in post-transplant WT versus TCF cKO cells (Fig 6E-G). Therefore, TCF-1

392 controls numerous immune genes and pathways in mature and alloactivated CD4 T cells, and

393 loss of this critical factor alters expression of these programs, ultimately affecting $\mathrm{T}$ cell function

394 and phenotype.

395 


\section{Discussion}

397 T Cell Factor-1 (TCF-1) is a T cell transcription factor that is known to be critical for T cell

398 development, activation, and in some cases, responses to pathogens (31). However, it unclear

399 whether TCF-1 may regulate alloactivated T cells during responses to alloantigen. TCF-1 is also

400 critical for supporting CD4 versus CD8 lineage maintenance in developing T cells $(6,32)$. It was

401 also unclear whether this lineage support is maintained by TCF-1 even in mature T cells $(33,34)$.

402 To address these gaps in knowledge, we studied loss of TCF-1 specifically in T cells during allo-

403 HSCT leading to GVHD. Using a unique mouse strain with T cell-specific deletion of TCF-1

404 (rather than global deletion), we were able to study the role of TCF-1 in mature and alloactivated

405 CD4 T cells (20). This manuscript, together with our companion manuscript, shows that TCF-1

406 does regulate mature CD4 and CD8 alloactivated T cells during GVHD (and GVL for CD8

407 cells). We discovered that TCF-1 appears to have a unique role in mature and alloactivated

408 CD4+ versus CD8+ T cells, potentially due to its ability to bind different lineage-specific genes

409 to help maintain identity in these disparate subsets.

410 Here, we showed that loss of TCF-1 in CD4 T cells leads to reduced severity and

411 persistence of GVHD symptoms. Tissue damage in the target organs (liver, skin, and small

412 intestine) was also reduced when donor cells were TCF-1-deficient. This suggests that TCF-1 is

413 indispensable for GVHD damage, and normally promotes damage to healthy host tissues driven

414 by donor $\mathrm{T}$ cells. T cell-mediated damage is a consequence of donor $\mathrm{T}$ cell migration,

415 proliferation, survival, and cytokine production. If any or all of these functions are disrupted,

416 then alloreactive T cells will be unable to induce GVHD. However, it is not ideal to fully disrupt

417 all of these functions, because the same processes also mediate protection against infection post-

418 transplant $(35,36)$. Here, we show that survival is reduced and exhaustion is increased in donor 
419 CD4 T cells from TCF-1-deficient mice. Interestingly, there appears to be a migration defect in

420 TCF-1 cKO donor T cells as well; however, given that chemokine and chemokine receptor

421 expression indicates that migration should be possible in these cells, it is more likely that this

422 apparent defect stems from a reduction in proliferation or survival.

423 During GVHD, production of cytokines by donor cells and host tissues causes damage to

424 nearby healthy host cells (37-39). Cytokine production was affected by loss of TCF-1, as donor

425 T cells from TCF cKO mice showed decreased production of TNF- $\alpha$, but not IFN- $\gamma$. When serum

426 from recipient mice was tested, there was an early (day 7) increase in production of TNF- $\alpha$ and

427 IFN- $\gamma$, as well as IL-2, suggesting that TCF cKO donor cells are initially highly activated. Later

428 on (day 14 and 21), levels of all three cytokines dropped. For IFN- $\gamma$, donor cell production was

429 not affected by loss of TCF-1, but serum production increased early on. This is likely from

430 increased production by host cells when in the presence of TCF cKO donor cells. This suggests

431 that despite early increased activation, cytokine production by TCF cKO donor cells quickly

432 reduces post-transplant, allowing disease to resolve.

433 We also found that TCF-1-deficient donor CD4 T cells are more exhausted in the liver

434 and trending towards more exhausted in the spleen at 7 days post-transplant than WT donor T

435 cells. Exhausted cells are unable to respond to antigen in the proper manner, so if the donor T

436 cells mediating GVHD damage become exhausted, they will no longer continue to cause

437 damage(40). Donor T cells must also proliferate during GVHD to replace effector cells, which

438 die quickly but drive damage to tissues (41). Proliferation of TCF cKO donor CD4 T cells was

439 also initially higher in spleen and liver of transplanted mice, which correlates with the increase in

440 serum IL-2. However, by day 14, the number of donor cells able to be sorted from recipient mice

441 is vastly reduced for TCF cKO donors compared to WT. Therefore, we hypothesize that despite 
442 the higher early activation and proliferation of TCF cKO T cells, these donor cells rapidly

443 become exhausted, stop proliferating, and die off. This would lead to reduced presence of donor

444 cells in the host after day 7, which correlates well with the observed drop in symptom severity

445 after the peak at day 7. Importantly, many of the functional changes we observed showed trends

446 rather than statistical significance between WT and TCF cKO donor cells. We expect that this is

447 because day 7 marks the beginning of the differences in disease outcome between groups, and

448 the differences become much more pronounced after this time point, as supported by GVHD

449 clinical scores and survival data. Therefore, we expect that as the disease outcomes separate

450 more between groups, the differences in $\mathrm{T}$ cell function would also become even more

451 significant. While TCF cKO donor cells may function similarly to WT cells early on, after day 7

452 they clearly begin to function differently, leading to changes in tissue damage and disease score.

453 We hypothesize that after day 7, TCF cKO donor T cells become exhausted and stop

454 proliferating and producing cytokines, allowing resolution of a usually persistent disease state 455 (41).

To examine the mechanism behind these functional changes, we phenotyped TCF cKO

457 and WT donor T cells prior to transplant. Despite having no effect on Eomes, T-bet, or CD122,

458 loss of TCF-1 led to increased polarization of CD44 expression. This manifested as expansion of

459 naive CD4 T cells, with a concomitant decrease in activating/transition CD4 T cells from the

460 TCF cKO donor mice. This suggests that TCF-1 normally promotes activation of T cells out of

461 the naive state for CD4 T cells, and loss of this factor allowed more cells to remain naïve (42).

462 TCF-1 is known to be highly expressed in naive T cells (34), and naive cells drive GVHD

463 damage $(15,43)$. Thus, it is clear that despite expansion of CD4 naive T cells in these TCF cKO

464 mice, functional and genetic changes to CD4 T cells caused by loss of TCF-1 prevent 
465

466

467

468

469

470

471

472

473

474

475

476

477

478

479

480

481

482

483

484

485

486

487

exacerbation of GVHD damage. These phenotypic effects were cell-intrinsic, indicating a direct

role of TCF-1 in controlling mature CD4 T cell phenotype. Thus, TCF-1 directly and cell-

intrinsically produces changes in CD4 T cell phenotype, which can impact alloactivated $\mathrm{T}$ cell

functioning. We also discovered that loss of TCF-1 altered expression of PD-1 and PD-L1 by

CD4 T cells following allotransplant. This suggests that the donor $\mathrm{T}$ cell suppressive profile is

altered following transplant. No changes were observed for CTLA-4, suggesting that TCR

signaling regulation is not disrupted by loss of TCF-1.

Finally, to examine what changes occurred to the genetic program following loss of TCF-

1, we performed RNA sequencing on pre- and post-transplant donor cells as an unbiased

approach. Gene expression by T cells changes drastically during alloactivation (44), and we

sought to understand how mature T cells lacking TCF-1 were different from those with TCF-1,

both before and after alloactivation. We FACS-sorted splenic donor CD4 T cells pre-transplant,

and both splenic and liver-derived donor cells post-transplanted (from recipients). We found that

many immune-related genes and pathways were altered by loss of TCF-1 in donor cells, both

pre- and post-transplant. Interestingly, allotransplantation appears to induce genetic programs

that are similar in both WT and TCF cKO donor cells, because the number of altered pathways

and genes when comparing strains was less for post-transplant cells than for pre-transplant cells.

This means that pre-transplant donor cells are more different based on expression of TCF-1 than

post-transplant cells are. Of note, the most significantly impacted pathway in post-transplant

cells (from spleen or liver) was cytokine/cytokine receptor interactions. This highlights the

importance of our finding that TCF cKO donor cells trend towards less TNF- $\alpha$ production,

suggesting that TNF- $\alpha$ is critical for driving GVHD damage (45), and therefore helping to

explain our observation that TCF cKO-transplanted mice have less severe GVHD. We also found 
488 that intestinal immunity and NOD signaling (46) were altered post-transplant, again helping to

489 explain the reduced gut symptoms seen in TCF cKO-transplanted mice (47). Alterations in the

490 chemokine signaling pathway were also detected, supporting our qPCR data showing that TCF-1

491 controls chemokine receptor expression on mature CD4 T cells $(48,49)$. Pre-transplant,

492 pathways such as cell cycle, T cell differentiation/signaling, and immune signaling pathways

493 were affected by loss of TCF-1, helping to explain the altered phenotype and function of mature

494 TCF cKO CD4 T cells (50). Overall, this sequencing data shows that TCF-1 is not only

495 important in T cell development, but is also critical for regulation of mature and alloactivated T

496 cells as well.

497 Our companion manuscript addresses the role of TCF-1 in mature alloactivated CD8 T

498 cells in this model. Interestingly, the effects on phenotype, gene expression changes, and

499 chemokine/chemokine receptor expression were different for CD8 versus CD4 T cells. The

500 ability of TCF-1 to maintain specific lineage traits for CD4 and CD8 T cells seems to be

501 maintained in mature alloactivated T cells as well, which was previously unknown. Overall, our

502 data show that TCF-1 directly regulates alloactivated mature CD4 T cells, which was previously

503 unknown. They also show that CD4 versus CD8 mature alloactivated T cells are both regulated

504 in different ways by TCF-1, leading to disparate effects of TCF-1 deficiency in these cells.

505 However, loss of TCF-1 in either cell type does produce a clinically optimal phenotype, with

506 reduced host tissue damage and GVHD severity over time. Thus, modulation of TCF-1 or critical

507 downstream factors may prove beneficial for reducing GVHD severity following allo-HSCT.

508 Additionally, TCF-1 modulation in T cells may be useful in other T cell-mediated disorders.

509

510

511

512 


\section{Materials and Methods}

515 Mouse Models. For transplant experiments, the following female donor mice were used: B6-

516 Ly5.1 (CD45.1+, "WT” or B6.SJL-Ptprca Pepcb /BoyCrl, 494 from Charles River), C57B1/6j

517 (CD45.2+, "WT”,", 000664 from Jackson Laboratories), Tcf7 flox x CD4cre (referred to here as

518 “TCF cKO”, obtained from Dr. Jyoti Misra Sen at at NIH by permission of Dr. Howard Xue,

519 and bred in-house (20), or CD4cre (022071 from Jackson Laboratories, bred in-house). These

520 donors were age-matched to each other and to recipients as closely as possible. Recipient mice

521 for transplant experiments were female BALB/c mice (CR:028 from Charles River, age 8 weeks

522 or older). Recipient mice for chimera experiments were Thy1.1 mice (B6.PL-Thy1a/CyJ, 000406

523 from Jackson Labs).

524

525 Bone Marrow Transplants. Short-term and long-term bone marrow transplant models were

526 employed. BALB/c recipient mice were irradiated twice with 400 cGy of $x$-rays (total dose 800

527 cGy), with a rest period of at least 12 hours between doses, and 4 hours of rest prior to

528 transplantation. T cells (total CD3+ or CD4+) were separated from WT, CD4cre, or TCF cKO

529 spleens using CD90.2 or CD4 microbeads and LS columns (Miltenyi, CD4: 130-117-043,

530 CD90.2: 130-121-278, LS: 130-042-401). The cells were then injected IV into the tail vein in

531 PBS. The recipient mice received $1 \times 10^{6} \mathrm{~T}$ cells per mouse, along with $10 \mathrm{X} 10^{6}$ bone marrow

532 cells collected from BALB/c mice. Bone marrow was T-cell depleted with CD90.2 MACS beads

533 (130-121-278 from Miltenyi) and LD columns (130-042-901 from Miltenyi). At day 7, the

534 recipients were humanely euthanized, and serum, spleen, lymph nodes, small intestine, skin, or

535 liver was collected, depending on the experiment. For migration experiments, the donor cells

536 were a 1:1 ratio of WT (CD45.1):WT(CD45.2), or WT:TCF cKO CD3 T cells (total 1 X10 ${ }^{6}$ 
537 cells). For GVHD experiments, the recipient mice were weighed and given a GVHD score from

538 day 1 to day 60, and group survival was analyzed. For mixed bone marrow chimera experiments,

539 a 1:4 ratio of WT (CD45.1) to TCF cKO (CD45.2) bone marrow (total 50x $10^{6}$ cells) was injected

540 into Thy 1.1 mice, and the mice were rested for 9 weeks. At 9 weeks post-transplant, tail vein

541 blood was collected and stained with anti-CD45.1 and anti-CD45.2 to detect the two donor cell

542 types. At 10 weeks, these chimeras were euthanized and their spleens were processed and stained

543 for phenotyping by flow cytometry.

544

545 Flow Cytometry, Sorting, and Phenotyping. For phenotyping experiments and pre-transplant

546 sorted cells, splenocytes were obtained from WT and TCF cKO mice. For all other experiments,

547 cells were obtained from transplanted recipients. Cells were incubated with RBC Lysis Buffer

548 (00-4333-57 from eBioscience) to remove red blood cells when necessary. After processing,

549 cells were stained in MACS buffer (1x PBS with EDTA and 4g/L BSA) with extracellular

550 markers, and were incubated for 30 minutes on ice. Cells were then washed and run on a BD

551 LSRFortessa flow cytometer to collect data. If intracellular markers were used, cells were

552 washed after extracellular staining, then fixed overnight using buffers from the Fix/Perm

553 Concentrate and Fixation Diluent from FOXP3 Transcription Factor Staining Buffer Set

554 (eBioscience cat. No. 00-5523-00). The following day, cells were washed in Perm buffer from

555 the same kit, and were stained with intracellular markers in Perm buffer for 40 minutes at room

556 temperature. Stained cells were resuspended in FACS buffer (eBioscience cat. No. 00-4222-26)

557 and transferred to flow tubes. All antibodies were used at 1:100 dilution, and were purchased

558 from Biolegend or eBioscience. The cells were then washed and run on a BD LSRFortessa. For

559 cell sorting, cells were stained in the same manner and run on a BD FACSAria, equipped with 
560 cold-sorting blocks. Cells were sorted into sorting media (50\% FBS in RPMI) for maximum

561 viability, or Trizol for RNAseq/qPCR experiments. All flow cytometry data was analyzed using

562 FlowJo software v9 (Treestar). Depending on the experiment, antibodies used were: anti-CD4

563 (FITC, PE, BV785, BV21), anti-CD8 (FITC, PE, APC, PerCP, Pacific Blue, PE/Cy7), anti-CD3

564 (BV605 or APC/Cy7), anti-H2Kb-Pacific Blue, anti-H2Kd-PE/Cy7, anti-CD122 (FITC or APC), 565 anti-CD44 (APC or Pacific Blue), anti-CD62L (APC/Cy7), anti-TNF- $\alpha$-FITC, anti-IFN- $\gamma$-APC, 566 anti-Eomes (AF488 or PE/Cy7), anti-T-bet-BV421, anti-CD45.2-PE/Dazzle594, anti-CD45.1-

567 APC, anti-Ki67 (PE or BV421), anti-PD-1-BV785, anti-CTLA-4-PE, and anti-PD-L1 (PE or

568 PE/Dazzle594), anti-Annexin V-FITC, LIVE/DEAD Near IR, anti-EdU-AF647 (click chemistry

569 kit), and anti-TOX-APC

570

571 Histology. At day 7, day 14, and day 21 post-transplant, organs were removed from WT or TCF

572 cKO CD4 T cell-transplanted recipient mice. The spleen, liver, small intestine, and skin from

573 back and ear were removed and fixed in $10 \%$ neutral buffered formalin. Tissue pieces were

574 sectioned and stained with hemotoxylin and eosin by the Histology Core at Cornell University

575 (https://www.vet.cornell.edu/animal-health-diagnostic-center/laboratories/anatomic-

576 pathology/services). Stained slides were then imaged and given a GVHD grade by a pathologist

577 at SUNY Upstate (A.M.) who was blinded to study conditions and slide identity. GVHD grade

578 was determined on a scale of 0-4 by the following criteria: for skin - basal cell vacuolation up to

579 frank epidermal denudation, for small intestine - single cell necrosis up to diffuse mucosal

580 denudation, and for liver - percent of bile duct showing epithelial damage (I $\leq 25 \%$, II $25-49 \%$,

581 III 50-74\%, IV 75-100\%). Links for grading criteria:

582 http://surgpathcriteria.stanford.edu/transplant/skinacutegvhd/printable.html, 
583 http://surgpathcriteria.stanford.edu/transplant/giacutegvhd/printable.html,

584 http://surgpathcriteria.stanford.edu/transplant/livergvhd/printable.html.

585

586 Isolation of lymphocytes from liver, small intestine, or lymph nodes. To isolate lymphocytes

587 from lymph nodes, the inguinal, brachial, and axillary lymph nodes were removed from

588 euthanized mice, and ground between glass slides to create a cell suspension. Ground cells were

589 rinsed into tubes using PBS and centrifuged to remove debris. To isolate lymphocytes from liver,

590 livers were perfused with 5-10mL of ice cold PBS to remove RBCs before organ removal. Livers

591 were then ground through a 70uM filter with PBS, centrifuged to remove debris, and

592 lymphocytes were isolated by a 22 minute spin in 40\% Percoll in RPMI/PBS (22 degrees C,

593 2200rpm, no brake, no acceleration). Isolated lymphocyte pellets were washed, lysed to remove

594 remaining RBCs, and resuspended with PBS or MACS buffer (BSA in PBS). To isolate

595 lymphocytes from small intestine, the intestine was removed and put in ice cold MACS buffer,

596 opened lengthwise, washed with MACS, and epithelial cells were stripped off by a 30 min

597 shaking incubation (37 C) in strip buffer (1x PBS, FBS, EDTA 0.5M, and DTT 1M). Guts were

598 then cut into small pieces and digested by a 30 min shaking incubation $(37 \mathrm{C})$ in digestion buffer

599 (collagenase, DNAse, and RPMI). The tubes were then vortexed, and liquid and solid gut pieces

600 were filtered through a 70uM filter to obtain a cell suspension. Percoll was then used to isolate

601 lymphocytes as for liver, with no RBC lysis afterwards. Gut cells were then placed in MACS

602 buffer for further use.

603

604 qPCR Analysis. Mice were short-term transplanted as described above (1X106 CD3 donor T

605 cells and $\left.10 \times 10^{6} \mathrm{BALB} / \mathrm{c} \mathrm{BM}\right)$, and at day 7, recipient mice were humanely euthanized. 
606 Splenocytes were obtained from pre- and post-transplanted mice, and FACS sorted as described

607 above to obtain donor cells. These cells were all sorted into Trizol, then RNA was extracted

608 using chloroform phase separation protocols (https://www.nationwidechildrens.org/

609 Document/Get/93327). The extracted RNA was eluted using the Qiagen RNEasy Minikit (74104

610 from Qiagen) and run on a spectrophotometer to determine concentration. RNA was then

611 converted to cDNA with the Invitrogen SuperScript IV First Strand Synthesis System kit

612 (18091050 from Invitrogen) and run on a spectrophotometer to determine concentration. Master

613 cocktail including 10ng/uL cDNA and Taqman Fast Advanced Master Mix (4444557 from

614 Invitrogen) was prepared for each sample, and 20uL was added to each well of a 96 well

615 TaqMan Array plate with chemokine/chemokine receptor primers (ThermoFisher, Mouse

616 Chemokines \& Receptors Array plate, 4391524). The plates were run on a Quantstudio 3

617 thermocycler according to manufacturer instructions for the TaqMan assay, and data were 618 analyzed using the Design and Analysis software v2.4 (provided by ThermoFisher). Five 619 separate recipient mice were sorted and cells were combined to make one sample for qPCR 620 testing per condition/organ.

621

622 Migration Assay. BALB/c mice were allotransplanted with 10x10 $10^{6}$ ALB/c BM, and a 1:1

623 mixture of WT CD45.1+ CD3 T cells with either WT CD45.2+ cells or TCF cKO CD45.2+ cells

624 (total $1 \times 10^{6}$ cells). This 1:1 mixture was checked prior to transplant to ensure a 1:1 ratio of CD4

625 to CD8 T cells per strain, as well as a 1:1 ratio of each donor type (WT:WT or WT:TCF cKO).

626 At day 7 post-transplant, recipient mice were euthanized and lymphocytes were obtained as

627 described above from spleen, lymph nodes, liver, and small intestine. The lymphocytes were

628 stained for $\mathrm{CD} 3, \mathrm{CD} 4, \mathrm{CD} 8$, and $\mathrm{H} 2 \mathrm{~Kb}$ to identify donor cells, as well as CD45.2 and CD45.1 to 
629 identify cells of each donor strain. The ratio of CD45.1 to CD45.2 cells (i.e. WT to WT or WT to

630 TCF cKO) was calculated from flow cytometry data and analyzed.

631

632 Cytokine Restimulation. Mice were short-term transplanted as described above (1.5x10 $\left.{ }^{6} \mathrm{CD} 3\right)$

633 donor T cells and 10x106 $\mathrm{BALB} / \mathrm{c} \mathrm{BM}$ ), and at day 7 post-transplant, recipient mice were

634 humanely euthanized and splenocytes were obtained. The splenocytes were cultured for 6 hours

635 at $37 \mathrm{C}$ and $7 \% \mathrm{CO} 2$ with GolgiPlug (1:1000) and PBS or anti-CD3 (1ug/mL)/anti-CD28

$636(2 \mathrm{ug} / \mathrm{mL})$ to restimulate them. After 6 hours, the cells were removed from culture, stained for

637 surface markers, fixed and permeabilized, then stained for the cytokines IFN-y and TNF-a using

638 the BD Cytokine Staining kit (555028), and run on a flow cytometer.

639

640

LEGENDplex Serum ELISA Assay. In addition to splenocytes, serum was collected from

641 recipient mice in the cytokine experiment, and analyzed using the Biolegend LEGENDplex

642 Assay Mouse Th Cytokine Panel kit (741043). This kit quantifies serum concentrations of: IL-2

643 (T cell proliferation), IFN-y and TNF-a (Th1 cells, inflammatory), IL-4, IL-5, and IL-13 (Th2

644 cells), IL-10 (Treg cells, suppressive), IL-17A/F (Th17 cells), IL-21 (Tfh cells), IL-22 (Th22

645 cells), IL-6 (acute/chronic inflammation/T cell survival factor), and IL-9 (Th2, Th17, iTreg, Th9

646 - skin/allergic/intestinal inflammation).

647

648 Proliferation Assay. Mice were short-term transplanted as described above (1x106 CD3 donor T 649 cells and $\left.10 \times 10^{6} \mathrm{BALB} / \mathrm{c} \mathrm{BM}\right)$, and recipient mice were injected at day 5-6 with 25mg/kg EdU 650 (20518 from Cayman Chemicals) in PBS. At day 7, the recipient mice were humanely

651 euthanized, and lymphocytes from spleen and liver were obtained. These cells were processed 
652 and stained using an EdU click chemistry kit (C10424 from Invitrogen), stained for H2Kb, CD3,

$653 \mathrm{CD} 4$, and CD8, and run on a flow cytometer. In vitro culture attempts mentioned as empirical

654 observations were done at $37 \mathrm{C}, 7 \% \mathrm{CO} 2$, with anti-CD3 (1ug/mL), anti-CD28 (2ug/mL), and in

655 some cases, IL-2 (1000IU/mL).

656

657 Death Assay. Mice were short-term transplanted as described above (1x10 ${ }^{6}$ CD3 donor T cells

658 and $\left.10 \times 10^{6} \mathrm{BALB} / \mathrm{c} \mathrm{BM}\right)$, and at day 7 post-transplant, cells from spleen and liver were stained

659 with Annexin V-FITC (V13242 from Invitrogen) and LIVE/DEAD Near IR (L34976 from

660 Invitrogen). Annexin V and NIR were used to identify dead (Ann.V+NIR+), live (Ann.V-NIR-),

661 and apoptotic (Ann.V+NIR-) cells. Donor T cells were identified by H2Kb, CD3, CD4, and

662 CD8.

663

664 Exhaustion/Activation Assay. Mice were short-term transplanted as described above (1X10

$665 \mathrm{CD} 3$ donor T cells and 10x10 $\left.10^{6} \mathrm{BLB} / \mathrm{c} \mathrm{BM}\right)$, and at day 7, lymphocytes were obtained from

666 spleens and livers of transplanted mice. The cells were stained with anti-TOX and anti-Ki67.

667 Donor T cells were identified by $\mathrm{H} 2 \mathrm{~Kb}+, \mathrm{CD} 3+, \mathrm{CD} 4+$, and $\mathrm{CD} 8+$.

668

669 Time course Assay. Mice were short-term transplanted as described above (1x106 CD4 donor T

670 cells and $\left.10 \times 10^{6} \mathrm{BALB} / \mathrm{c} \mathrm{BM}\right)$, and at day 7 , day 14 , and day 21 , lymphocytes were obtained

671 from spleens and livers of transplanted mice. The cells were stained with anti-PD-1, anti-PD-L1,

672 and anti-CTLA4. Donor T cells were identified by $\mathrm{H} 2 \mathrm{~Kb}+, \mathrm{CD} 3+, \mathrm{CD} 4+$, and CD8+. 
674 cDNA Extraction and PCR. Donor mice were genotyped using PCR. Ear punches were taken

675 from each mouse at 4 weeks of age, DNA was extracted, and run in a PCR reaction using the 676 Accustart II Mouse Genotyping kit (95135-500 from Quanta Biosciences). Standard PCR

677 reaction conditions and primer sequences from Jackson Laboratories were used for Eomes, T-

678 bet, and CD4cre. For Tcf7, primer sequences and reaction conditions were obtained from Dr.

679 Jyoti Misra Sen of NIH.

680

681

RNA Sequencing. Mice were short-term transplanted as described above $\left(1 \times 10^{6}\right.$ CD3 donor T

682 cells and $\left.10 \times 10^{6} \mathrm{BALB} / \mathrm{c} \mathrm{BM}\right)$, and at day 7, recipient mice were humanely euthanized.

683 Splenocytes were obtained from pre- and post-transplanted mice, and liver cells were obtained

684 from post-transplant mice only. For TCF cKO-transplanted mice, cells were also sorted from

685 recipients on day 14. Cells were FACS sorted as described above to obtain donor cells. These

686 cells were all sorted into Trizol and brought to the Molecular Analysis Core at SUNY Upstate

687 (https://www.upstate.edu/research/facilities/molecular-analysis.php) for RNA extraction and

688 library prep, followed by RNA sequencing analysis at the University at Buffalo Genomics Core

689 (http://ubnextgencore.buffalo.edu). Data were provided and were analyzed using Partek Flow

690 software. This data will be deposited at (https://www.ncbi.nlm.nih.gov/geo/).

691

692

693 Statistical Analysis. All numerical data is reported as means with standard deviation unless

694 otherwise noted. Data was analyzed for significance with GraphPad Prism v7 or v9. Differences

695 were determined using one-way or two-way ANOVA and Tukey's multiple comparisons tests

696 for three or more groups, or with a student's t-test when only two groups were used. Kaplan- 
697 Meier survival analysis was used for survival experiments. Chi-square analysis was used for

698 GVHD histology grades. All tests were two-sided. P-values less than or equal to 0.05 were

699 considered significant. All transplant experiments were done with N=3-5 mice per group, and

700 repeated at least twice. Ex vivo experiments were done two to three times unless otherwise noted

701 with at least three replicates per condition each time. RNAseq was done once with three

702 replicates per group. qPCR was done once with one sample per condition, and 5 mice combined

703 to make the one sample. Sorting was done once for each of these two experiments, and data were

704 recorded for Supp. Fig. 4. The time course assay was done once with 4-6 samples per group. The

705 chimera experiment was done once with 5 chimeras ( 5 samples per donor type). Data in figures

706 are presented as mean and SD unless otherwise noted.

707

708 Study Approval. All animal studies were reviewed and approved by the IACUC at SUNY

709 Upstate Medical University. All procedures and experiments were performed according to these

710 approved protocols.

711

712

713

714

715

716

717

718

719

720

721

722

723

724

725

726

727

728 


\section{$\underline{\text { Author Contributions }}$}

731 RH designed and conducted experiments, analyzed data, and wrote the manuscript. MK assisted

732 with scientific/technical research design, and edited the manuscript. MM assisted with data

733 collection. QY provided technical and scientific advice and assisted with data analysis. AM

734 performed blinded histology analyses. JMS provided TCF cKO mice with permission of Howard

735 Xue and helped edit the manuscript. IF produced gene expression heatmaps for RNAseq data.

736

737 Acknowledgments

738 We thank all members of the Karimi lab for helpful discussions. This research was funded in part

739 by a grant from the National Blood Foundation Scholar Award to MK, the National Institutes of

740 Health (NIH LRP \#L6 MD0010106 and K22 (AI130182) to MK), and an Upstate Medical

741 University Cancer Center grant (1146249-1-75632) to MK. JMS was supported by the

742 Intramural Research Program of the National Institute of Aging

743 We thank Dr. Howard Xue for permission to use TCF cKO mice. Flow cytometers and sorters

744 were provided and maintained by Lisa Phelps of the SUNY Upstate Flow Core. RNA extraction

745 and prep was done by Karen Gentile of the SUNY Upstate Molecular Analysis core, and RNA

746 sequencing was done by the University at Buffalo Genomics Core.

747

748

749

750

751

752

753 


\section{References}

755

756

757

758

759

760

761

762

763

764

765

766

767

768

769

770

771

772

773

774

775

776

777

778

779

780

781

782

783

784

785

786

787

788

789

790

791

792

793

794

795
1. Ferrara JL. Blood and Marrow Transplant Clinical Trials Network: Progress since the State of the Science Symposium 2007. Biol Blood Marrow Transplant. 2014;20(2):14953.

2. Geerman S, and Nolte MA. Impact of T cells on hematopoietic stem and progenitor cell function: Good guys or bad guys? World J Stem Cells. 2017;9(2):37-44.

3. Murray J, Stringer J, and Hutt D. In: Kenyon M, and Babic A eds. The European Blood and Marrow Transplantation Textbook for Nurses: Under the Auspices of EBMT. Cham (CH); 2018:221-51.

4. Blazar BR, Murphy WJ, and Abedi M. Advances in graft-versus-host disease biology and therapy. Nat Rev Immunol. 2012;12(6):443-58.

5. Yu J, Parasuraman S, Shah A, and Weisdorf D. Mortality, length of stay and costs associated with acute graft-versus-host disease during hospitalization for allogeneic hematopoietic stem cell transplantation. Curr Med Res Opin. 2019;35(6):983-8.

6. Yu Q, Erman B, Park JH, Feigenbaum L, and Singer A. IL-7 receptor signals inhibit expression of transcription factors TCF-1, LEF-1, and RORgammat: impact on thymocyte development. J Exp Med. 2004;200(6):797-803.

7. Wang F, Qi Z, Yao Y, Yu G, Feng T, Zhao T, et al. Exploring the stage-specific roles of Tcf-1 in T cell development and malignancy at single-cell resolution. Cell Mol Immunol. 2021;18(3):644-59.

8. Jeannet G, Boudousquie C, Gardiol N, Kang J, Huelsken J, and Held W. Essential role of the Wnt pathway effector Tcf-1 for the establishment of functional CD8 T cell memory. Proc Natl Acad Sci U S A. 2010;107(21):9777-82.

9. DeWolf $S$, and Sykes M. Alloimmune T cells in transplantation. J Clin Invest. 2017;127(7):2473-81.

10. Zhou X, Yu S, Zhao DM, Harty JT, Badovinac VP, and Xue HH. Differentiation and persistence of memory CD8(+) T cells depend on T cell factor 1 . Immunity. 2010;33(2):229-40.

11. Paley MA, and Wherry EJ. TCF-1 flips the switch on Eomes. Immunity. 2010;33(2):145-7.

12. Chen Z, Ji Z, Ngiow SF, Manne S, Cai Z, Huang AC, et al. TCF-1-Centered Transcriptional Network Drives an Effector versus Exhausted CD8 T Cell-Fate Decision. Immunity. 2019;51(5):840-55 e5.

13. Pearce EL, Mullen AC, Martins GA, Krawczyk CM, Hutchins AS, Zediak VP, et al. Control of effector CD8+ T cell function by the transcription factor Eomesodermin. Science. 2003;302(5647):1041-3.

14. Fu J, Wang D, Yu Y, Heinrichs J, Wu Y, Schutt S, et al. T-bet is critical for the development of acute graft-versus-host disease through controlling $T$ cell differentiation and function. J Immunol. 2015;194(1):388-97.

15. Mammadli M, Huang W, Harris R, Sultana A, Cheng Y, Tong W, et al. Targeting Interleukin-2-Inducible T-Cell Kinase (ITK) Differentiates GVL and GVHD in Allo-HSCT. Front Immunol. 2020;11:593863. 
801

802

803

804

805

806

807

808

809

810

811

812

813

814

815

816

817

818

819

820

821

822

823

824

825

826

827

828

829

830

831

832

833

834

835

836

837

838

839

16. Germar K, Dose $\mathrm{M}$, Konstantinou T, Zhang J, Wang $\mathrm{H}$, Lobry $\mathrm{C}$, et al. T-cell factor 1 is a gatekeeper for T-cell specification in response to Notch signaling. Proc Natl Acad Sci U S A. 2011;108(50):20060-5.

17. Sharma A, Berga-Bolanos R, and Sen JM. T cell factor-1 controls the lifetime of CD4+ $\mathrm{CD} 8+$ thymocytes in vivo and distal $\mathrm{T}$ cell receptor alpha-chain rearrangement required for NKT cell development. PLoS One. 2014;9(12):e115803.

18. Okamura RM, Sigvardsson M, Galceran J, Verbeek S, Clevers H, and Grosschedl R. Redundant regulation of $\mathrm{T}$ cell differentiation and TCRalpha gene expression by the transcription factors LEF-1 and TCF-1. Immunity. 1998;8(1):11-20.

19. Yu S, Zhou X, Steinke FC, Liu C, Chen SC, Zagorodna O, et al. The TCF-1 and LEF-1 transcription factors have cooperative and opposing roles in $\mathrm{T}$ cell development and malignancy. Immunity. 2012;37(5):813-26.

20. Steinke FC, Yu S, Zhou X, He B, Yang W, Zhou B, et al. TCF-1 and LEF-1 act upstream of Th-POK to promote the CD4(+) T cell fate and interact with Runx3 to silence Cd4 in CD8(+) T cells. Nat Immunol. 2014;15(7):646-56.

21. Verbeek S, Izon D, Hofhuis F, Robanus-Maandag E, te Riele $H$, van de Wetering $M$, et al. An HMG-box-containing T-cell factor required for thymocyte differentiation. Nature. 1995;374(6517):70-4.

22. Ioannidis $\mathrm{V}$, Beermann $\mathrm{F}$, Clevers $\mathrm{H}$, and Held W. The beta-catenin--TCF-1 pathway ensures CD4(+)CD8(+) thymocyte survival. Nat Immunol. 2001;2(8):691-7.

23. Reddy P, and Ferrara JLM. StemBook. Cambridge (MA); 2008.

24. Cooke KR, Kobzik L, Martin TR, Brewer J, Delmonte J, Jr., Crawford JM, et al. An experimental model of idiopathic pneumonia syndrome after bone marrow transplantation: I. The roles of minor H antigens and endotoxin. Blood. 1996;88(8):32309.

25. Coghill JM, Sarantopoulos S, Moran TP, Murphy WJ, Blazar BR, and Serody JS. Effector CD4+ T cells, the cytokines they generate, and GVHD: something old and something new. Blood. 2011;117(12):3268-76.

26. Pidala J, Kim J, Anasetti C, Nishihori T, Betts B, Field T, et al. The global severity of chronic graft-versus-host disease, determined by National Institutes of Health consensus criteria, is associated with overall survival and non-relapse mortality. Haematologica. 2011;96(11):1678-84.

27. Clinical manifestations, diagnosis, and grading of acute graft-versus-host disease

28. Johnson JL, Georgakilas G, Petrovic J, Kurachi M, Cai S, Harly C, et al. LineageDetermining Transcription Factor TCF-1 Initiates the Epigenetic Identity of T Cells. Immunity. 2018;48(2):243-57 e10.

29. Ferrara JL, Levine JE, Reddy P, and Holler E. Graft-versus-host disease. Lancet. 2009;373(9674):1550-61.

30. Riley JL. PD-1 signaling in primary T cells. Immunol Rev. 2009;229(1):114-25.

31. Garcia-Perez L, Famili F, Cordes M, Brugman M, van Eggermond M, Wu H, et al. Functional definition of a transcription factor hierarchy regulating $T$ cell lineage commitment. Sci Adv. 2020;6(31):eaaw7313.

32. Raghu $\mathrm{D}, \mathrm{Xue} \mathrm{HH}$, and Mielke LA. Control of Lymphocyte Fate, Infection, and Tumor Immunity by TCF-1. Trends Immunol. 2019;40(12):1149-62. 
840

841

842

843

844

845

846

847

848

849

850

851

852

853

854

855

856

857

858

859

860

861

862

863

864

865

866

867

868

869

870

871

872

873

874

875

876

877

878

879

880

881

882

883

33. Ye Z, Gould TM, Zhang H, Jin J, Weyand CM, and Goronzy JJ. The GSK3beta-beta-cateninTCF1 pathway improves naive T cell activation in old adults by upregulating miR-181a. NPJ Aging Mech Dis. 2021;7(1):4.

34. Yu Q, Sharma A, and Sen JM. TCF1 and beta-catenin regulate T cell development and function. Immunol Res. 2010;47(1-3):45-55.

35. Biernacki MA, Sheth VS, and Bleakley M. T cell optimization for graft-versus-leukemia responses. JCI Insight. 2020;5(9).

36. Briones J, Novelli S, and Sierra J. T-cell costimulatory molecules in acute-graft-versus host disease: therapeutic implications. Bone Marrow Res. 2011;2011:976793.

37. Henden AS, and Hill GR. Cytokines in Graft-versus-Host Disease. J Immunol. 2015;194(10):4604-12.

38. Holler E. Cytokines, viruses, and graft-versus-host disease. Curr Opin Hematol. 2002;9(6):479-84.

39. Kumar S, Mohammadpour H, and Cao X. Targeting Cytokines in GVHD Therapy. J Immunol Res Ther. 2017;2(1):90-9.

40. Khan O, Giles JR, McDonald S, Manne S, Ngiow SF, Patel KP, et al. TOX transcriptionally and epigenetically programs CD8(+) T cell exhaustion. Nature. 2019;571(7764):211-8.

41. Hill GR, and Koyama M. Cytokines and costimulation in acute graft-versus-host disease. Blood. 2020;136(4):418-28.

42. Welten SPM, Yermanos A, Baumann NS, Wagen F, Oetiker N, Sandu I, et al. Tcf1(+) cells are required to maintain the inflationary $T$ cell pool upon MCMV infection. Nat Commun. 2020;11(1):2295.

43. Dutt S, Baker J, Kohrt HE, Kambham N, Sanyal M, Negrin RS, et al. CD8+CD44(hi) but not CD4+CD44(hi) memory T cells mediate potent graft antilymphoma activity without GVHD. Blood. 2011;117(11):3230-9.

44. Conley JM, Gallagher MP, and Berg L. T Cells and Gene Regulation: The Switching On and Turning Up of Genes after T Cell Receptor Stimulation in CD8 T Cells. Front Immunol. 2016;7:76.

45. Mancusi A, Piccinelli S, Velardi A, and Pierini A. The Effect of TNF-alpha on Regulatory T Cell Function in Graft-versus-Host Disease. Front Immunol. 2018;9:356.

46. Heim VJ, Stafford CA, and Nachbur U. NOD Signaling and Cell Death. Front Cell Dev Biol. 2019;7:208.

47. Penack O, Smith OM, Cunningham-Bussel A, Liu X, Rao U, Yim N, et al. NOD2 regulates hematopoietic cell function during graft-versus-host disease. J Exp Med. 2009;206(10):2101-10.

48. Castor MG, Pinho V, and Teixeira MM. The role of chemokines in mediating graft versus host disease: opportunities for novel therapeutics. Front Pharmacol. 2012;3:23.

49. Bouazzaoui A, Spacenko E, Mueller G, Miklos S, Huber E, Holler E, et al. Chemokine and chemokine receptor expression analysis in target organs of acute graft-versus-host disease. Genes Immun. 2009;10(8):687-701.

50. Yakoub-Agha I, Saule P, Depil S, Micol JB, Grutzmacher C, Boulanger-Villard F, et al. A high proportion of donor CD4+ T cells expressing the lymph node-homing chemokine receptor CCR7 increases incidence and severity of acute graft-versus-host disease in patients undergoing allogeneic stem cell transplantation for hematological malignancy. 
Wnt $\beta$-catenin pathway

890

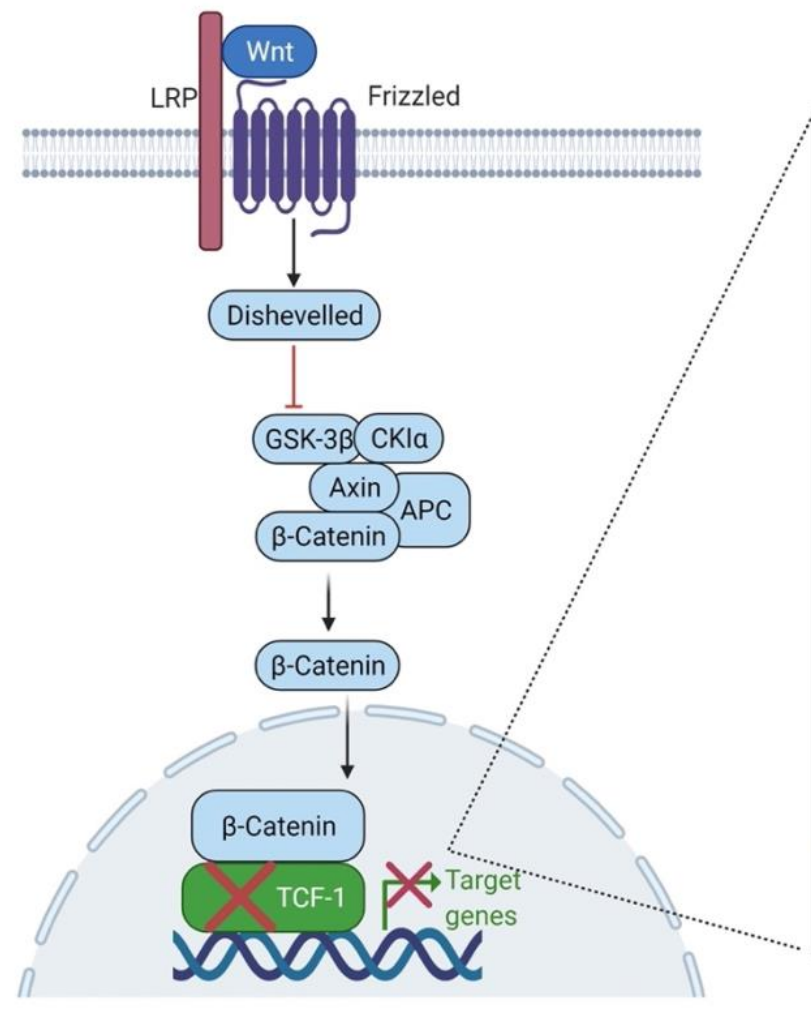

CD4+ Donor T Cells

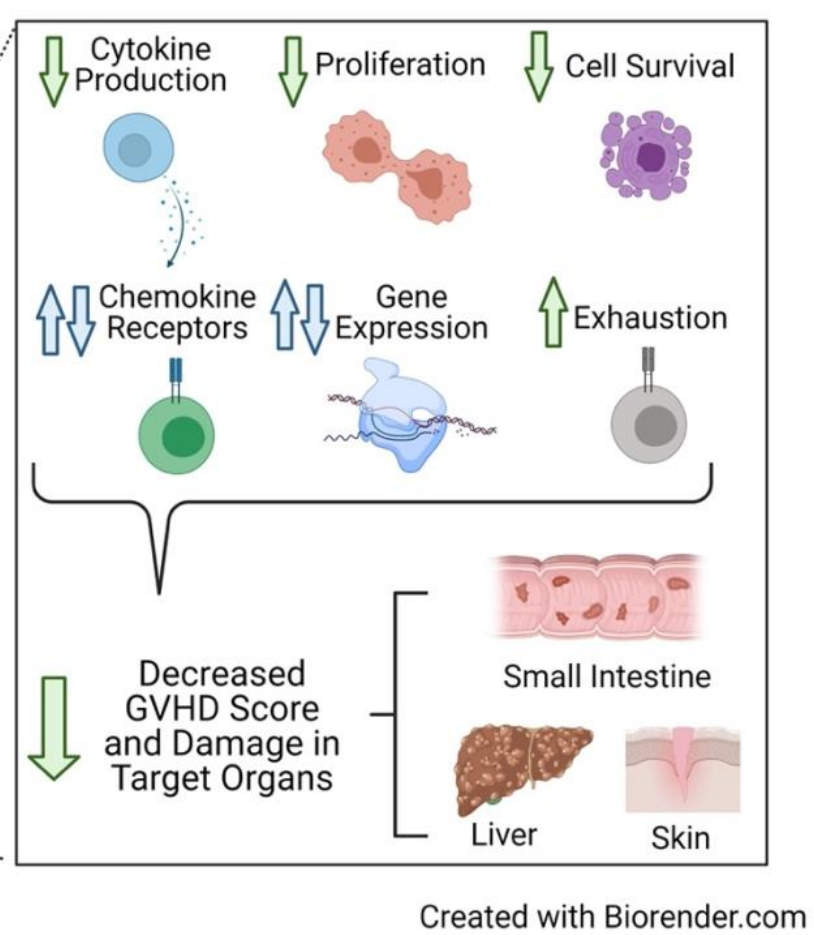

891

Summary Figure: TCF-1 regulates GVHD severity and persistence by controlling mature

892

893

894

895

896

897

\section{alloactivated CD4 $\mathrm{T}$ cell functions and gene expression. T Cell Factor-1 (TCF-1) is a Wnt}

pathway transcription factor found in T cells. Loss of this factor specifically in $\mathrm{T}$ cells reduces

GVHD severity and persistence due to changes in T cell function, including: decreased cytokine production, proliferation, and cell survival; increased exhaustion; and altered gene expression and expression of chemokine receptors. These changes lead to reduced GVHD score and less damage to target organs, including small intestine, liver, and skin. 
898

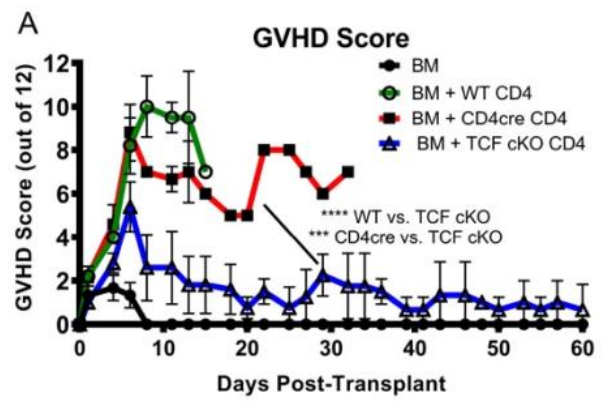

C

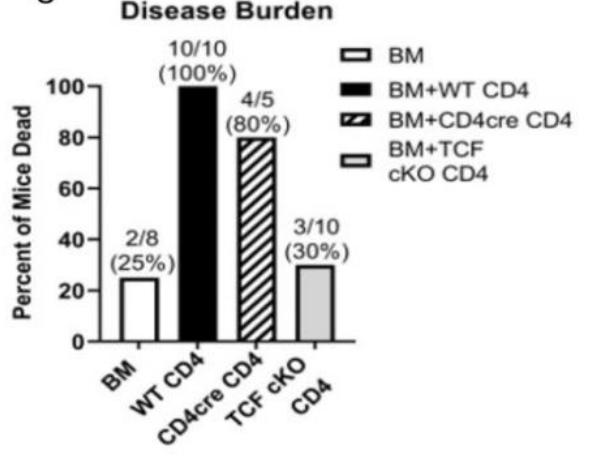

B

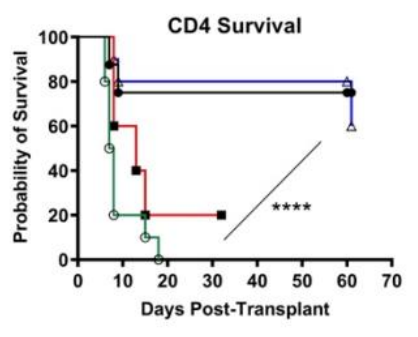

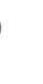

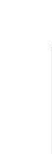

D

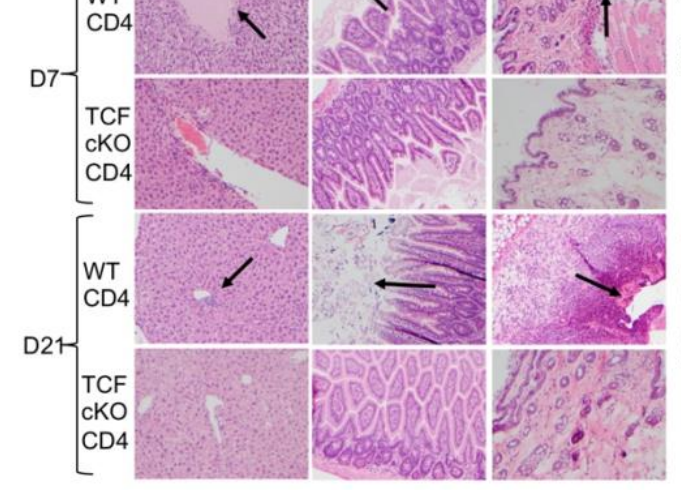

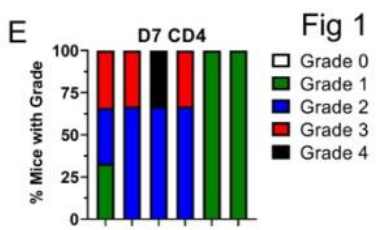
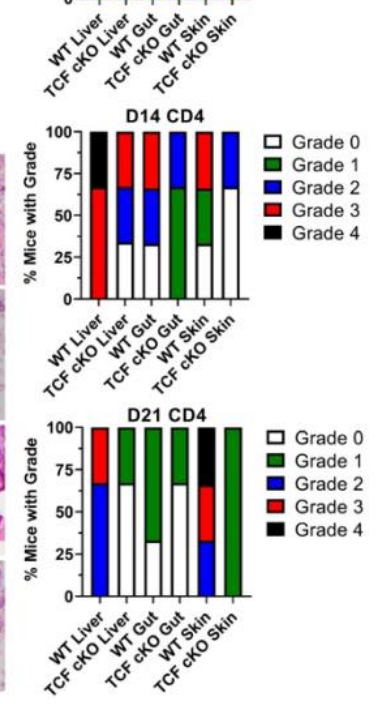

Figure Legends:

Figure 1: Loss of TCF-1 in donor CD4 T cells reduces severity and persistence over time of

901 GVHD symptoms. Recipient BALB/c mice (MHC haplotype D) were lethally irradiated and

902 allotransplanted with $10 \times 10^{6} \mathrm{BALB} / \mathrm{c}$ bone marrow (BM) cells and $1 \times 10^{6} \mathrm{CD} 4 \mathrm{~T}$ cells from

903 WT, CD4cre, or TCF cKO donor mice (C57Bl6 background, MHC haplotype B). The mice were

904 weighed and given a GVHD clinical score three times per week for 60 days. Score was

905 determined by combined scores for fur texture, activity level, posture, skin integrity, weight loss,

906 and diarrhea. (A) Clinical scores for mice over 60 days. Mean and SD plotted, analyzed by two-

907 way ANOVA. (B) Survival of mice in each group over the 60 day experiment, analyzed with

908 Kaplan-Meier survival statistics. (C) Disease burden as determined by percent of mice in each

909 group that died from GVHD by the end of the experiment. Number of mice (dead and total) per

910 group as well as percent dead are noted above each bar. (D) Organs (skin, liver, and small

911 intestine AKA “gut") from recipient mice taken at day 7, day 14, and day 21 post-transplant, 
912 sectioned, and stained with H\&E. A pathologist (A.M.) who was blinded to study conditions and

913 sample groups was given deidentified slides, and gave each tissue section a GVHD grade based

914 on damage and other indicators (detailed further in Methods). Shown here are representative

915 sections from D7 and D21 for each organ. Arrows indicate areas of damage or lymphocytic

916 infiltration. (E) Histology GVHD grades were graphed as percentage of mice in each group with

917 that grade. Grades 0-2 are considered mild GVHD and grades 3-4 are considered severe GVHD.

918 Chi-square analyses were used to determine differences between grade frequencies. **** means

919 p-value $\leq$ 0.0001. N=3-5 per group for A-B with one representative experiment shown, $\mathrm{N}=5-10$

920 per group in $\mathrm{C}$ with two experiments combined, $\mathrm{N}=3$ per group for $\mathrm{D}-\mathrm{E}$, one representative photo

921 shown and summary data in E.

922
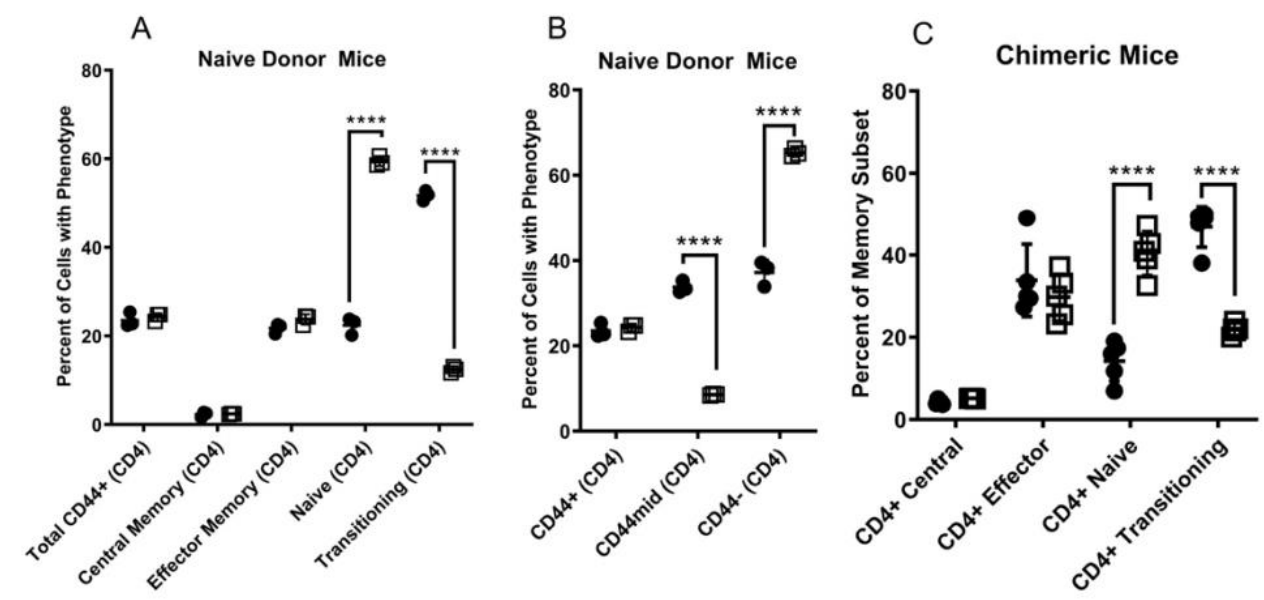

Fig.2

923

924

Figure 2: Loss of TCF-1 drives changes to mature CD4 T cell memory identity which are

925

primarily cell-intrinsic. (A-B) Naive WT or TCF cKO donor mice were euthanized,

926

splenocytes were obtained and stained for flow cytometry, and run on a BD LSRFortessa flow

927 cytometer. (A) Percent of CD4 T cells expressing naive, transitioning/activating, central

928 memory, or effector memory phenotypes. (B) Percent of CD4 T cells expressing CD44. (C-D) 
929 Thy1.1 mice were lethally irradiated and reconstituted with a 1:4 (WT:TCF cKO) mixture of

930 bone marrow cells. At 9 weeks, blood was checked by flow cytometry to ensure reconstitution,

931 and at 10 weeks, flow cytometry phenotyping was performed. WT donor cells were identified by

932 CD45.1, while TCF cKO donor cells were identified by CD45.2 (C) Percent of chimeric CD4 T

933 cells expressing naive, transitioning/activating, central memory, or effector memory phenotypes.

934 (D) Percent of chimeric CD4 T cells expressing CD122. All data are shown as individual points

935 with mean and SD, all data were analyzed with Student's t-test, one-way ANOVA, or two-way

936 ANOVA (depending on data groups), **** means p-value $\leq 0.0001$, and $* * *$ means $\mathrm{p}$-value $\leq$

937 0.001. For $A-B, N=3$ per group with one representative experiment shown, for $C-D, n=5$ per

938 group, one experiment shown (done once).

Fig.3

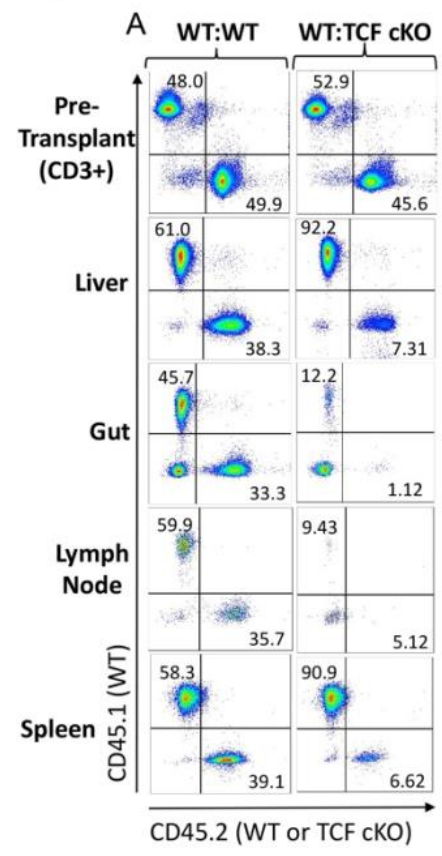

B
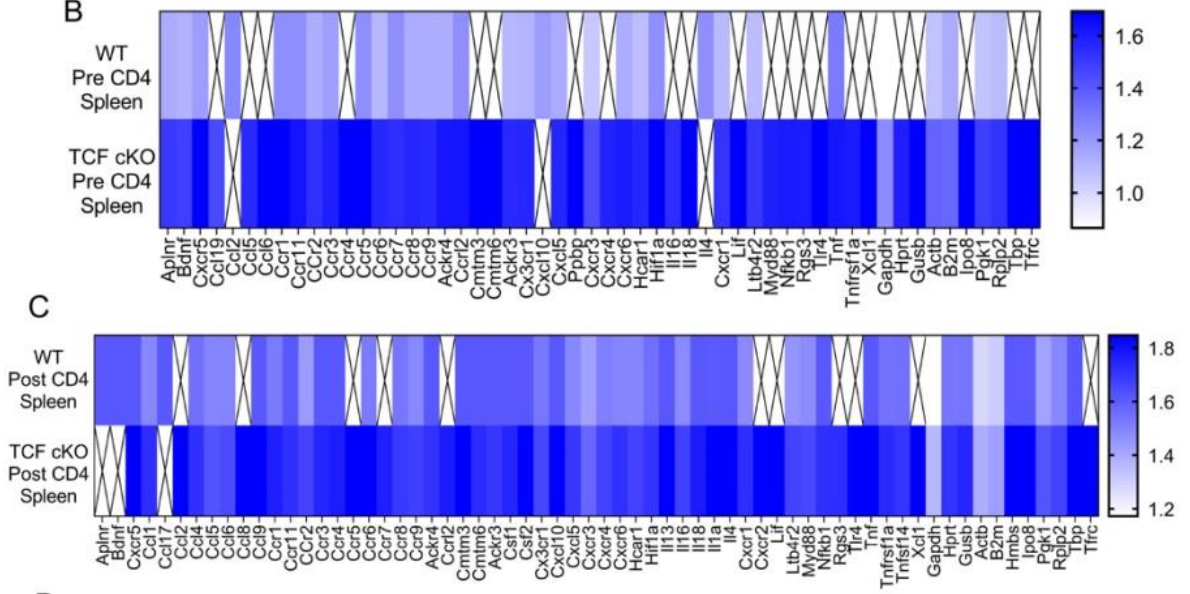

D

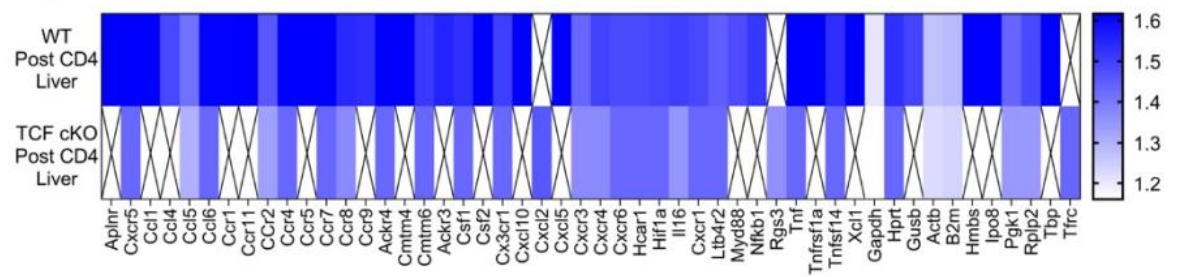

941 Figure 3: Loss of TCF-1 induces an apparent migration defect and changes to

942 chemokine/chemokine receptor expression in mature CD4 T cells during alloactivation. 
943 Recipient BALB/c mice were lethally irradiated and allotransplanted with $10 \mathrm{X} 10^{6} \mathrm{BALB} / \mathrm{c}$ bone 944 marrow $(\mathrm{BM})$ cells and $1 \times 10^{6} \mathrm{CD} 3 \mathrm{~T}$ cells. The $\mathrm{T}$ cells were a 1:1 mixture of CD3 T cells from 945 WT (CD45.1) and TCF cKO (CD45.2) donor mice, or WT (CD45.1) and WT (CD45.2) control 946 mice. (A) Prior to transplant, the cell mixture was checked to ensure a 1:1 ratio of CD4/CD8 T 947 cells, and a 1:1 ratio of each donor strain (using CD45.1 and CD45.2). At day 7 post-transplant, 948 the spleen, liver, small intestine ("gut"), and lymph nodes were taken from euthanized recipient 949 mice and processed to obtain lymphocytes. The cells were then stained for $\mathrm{H} 2 \mathrm{~Kb}$ (donor cells), $950 \mathrm{H} 2 \mathrm{KD}$ (recipient cells), CD3, CD4, CD8, CD45.1, and CD45.2. The pre-transplant flow plots are 951 shown on top, and one representative post-transplant flow plot for WT:WT or WT:TCF cKO 952 mice is shown per organ below. (B-D) To perform qPCR, BALB/c mice were lethally irradiated 953 and allotransplanted with $1 \times 10^{6} \mathrm{CD} 3$ donor cells and BALB/c BM, as above. Donor CD4 T cells 954 from WT and TCF cKO mice were FACS sorted both pre-transplant and 7 days post-transplant, 955 from spleen (both pre and d7) and liver ( $\mathrm{d} 7$ only). The cells were sorted in Trizol, and RNA was 956 extracted using chloroform and converted into cDNA using a synthesis kit (more details in 957 Methods). cDNA was added to premade mouse chemokine/chemokine receptor primer plates 958 (ThermoFisher), and run on a QuantStudio 3 thermocycler. Results are shown as a heatmap of 959 fold change per gene, compared to 18 S reference gene for each plate. Boxes with an "X" 960 represent signals too low to detect or otherwise unreadable due to technical error. Heatmaps 961 compare WT versus TCF cKO CD4 T cells in (B) pre-transplant spleen, (C) post-transplant 962 spleen, and (D) post-transplant liver. $\mathrm{N}=3$ per group for A with one representative experiment 963 shown, $\mathrm{N}=5$ mice into one sample per condition for B-D, summary data shown. 
- WT

口 TCF cKO

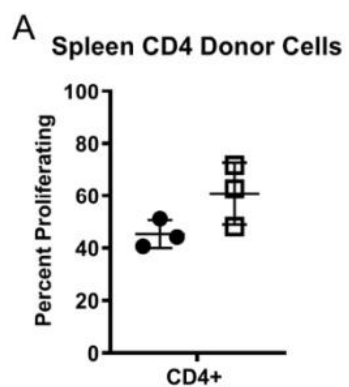

B
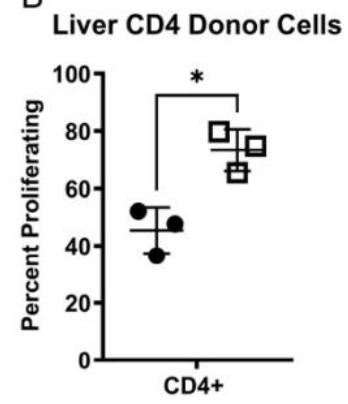
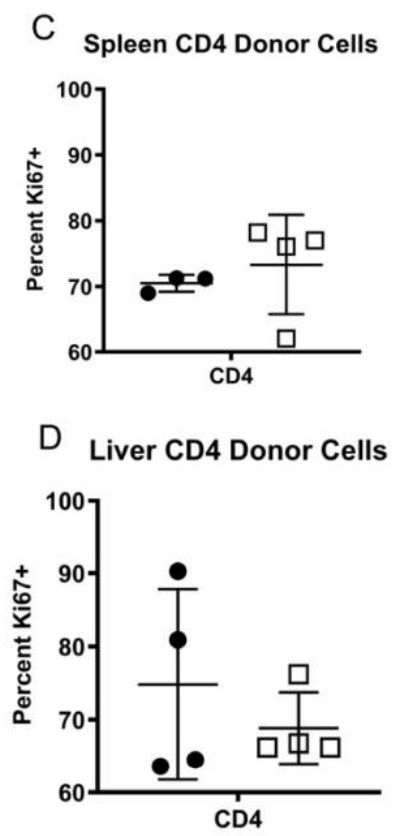

964

965

966

967

968

969

970

971

972

973 cells were stained for $\mathrm{H} 2 \mathrm{~Kb}, \mathrm{CD} 3, \mathrm{CD} 4, \mathrm{CD} 8$, and Ki67 to identify proliferating/activated cells 974 in (C) spleen and (D) liver. (E-F) Recipient mice were allotransplanted as before, and at day 7,
975 spleens and livers were taken from euthanized recipients. Lymphocytes were isolated and stained 974 in (C) spleen and (D) liver. (E-F) Recipient mice were allotransplanted as before, and at day 7,
975 spleens and livers were taken from euthanized recipients. Lymphocytes were isolated and stained 976 for CD3, CD4, CD8, H2Kb, and with Annexin V-FITC and LIVE/DEAD Near IR. Cells were

Figure 4: TCF-1 controls proliferation, apoptosis, and exhaustion of mature alloactivated

CD4 T cells. (A-B) Recipient BALB/c mice were allotransplanted with BALB/c BM and 1 X10

WT or TCF cKO donor CD3 T cells, as before. On day 5 and 6, recipient mice were injected i.p. with $25 \mathrm{mg} / \mathrm{kg}$ of EdU in PBS. At day 7, spleens and livers were removed from euthanized recipient mice, lymphocytes were isolated, and stained for EdU with a click chemistry kit. Cells were also stained for $\mathrm{H} 2 \mathrm{~Kb}, \mathrm{CD} 3, \mathrm{CD} 4$, and CD8. Graphs show percent proliferating (by EdU+) in (A) spleen and (B) liver. (C-D) Recipient mice were allotransplanted as before, and at day 7 spleens and livers were obtained from euthanized recipients. Lymphocytes were isolated, and
Fig.4
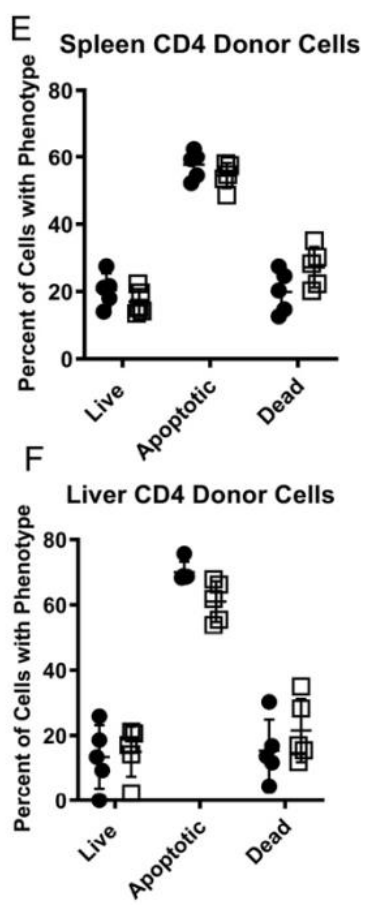

G Spleen CD4 Donor Cells

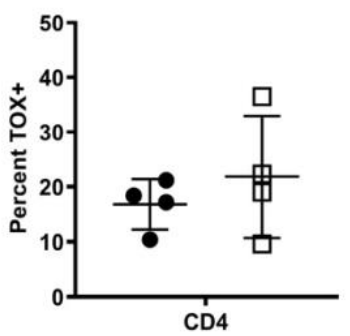

H Liver CD4 Donor Cells

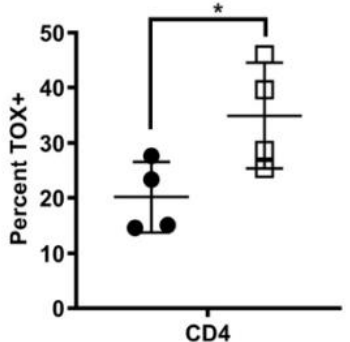


977 identified as live (Ann.V-IR-), apoptotic(Ann.V+IR-), or dead (Ann.V+IR+), in both (E) spleen

978 and (F) liver. (G-H) Recipient mice were allotransplanted as before, and at day 7 spleens and

979 livers were obtained from euthanized recipients. Cells were stained for H2Kb, CD3, CD4, and

980 CD8, then fixed/permeabilized and stained for TOX to identify exhausted cells in (G) spleen and

981 (H) liver. All data are shown as individual points with mean and SD, were analyzed with one-

982 way or two-way ANOVA, and * means $\mathrm{p}$-value $\leq 0.05 . \mathrm{N}=3-5$ per group with one representative

983 experiment shown.

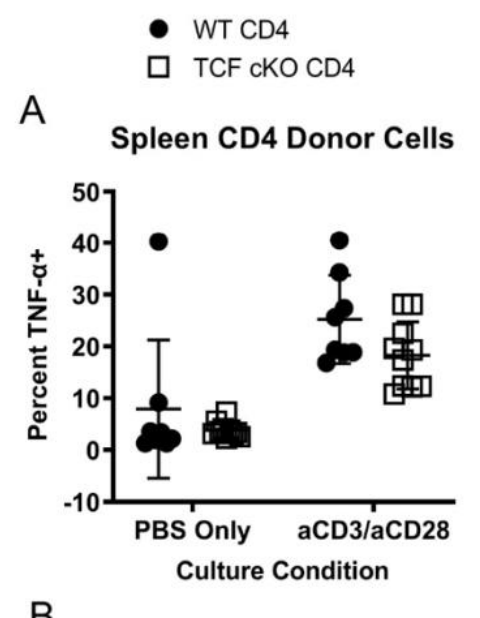

B

Spleen CD4 Donor Cells

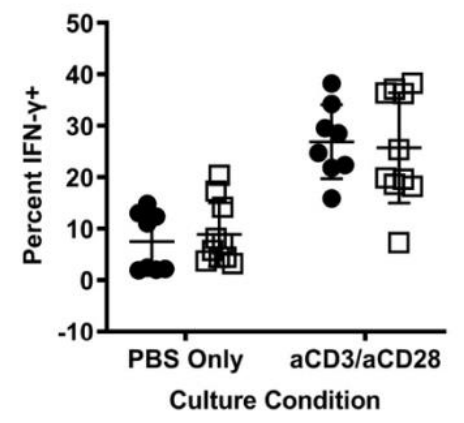

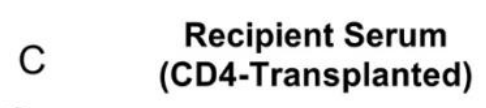

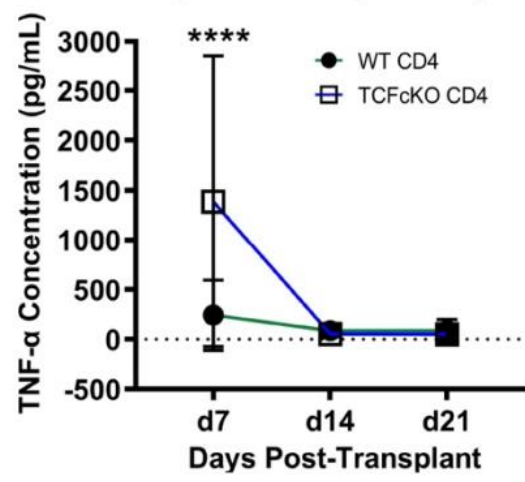

D
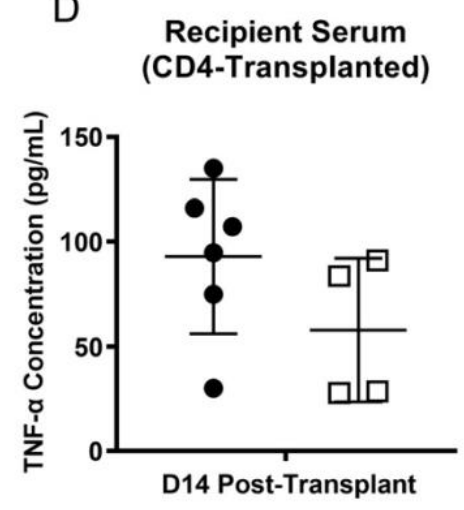

Fig.5
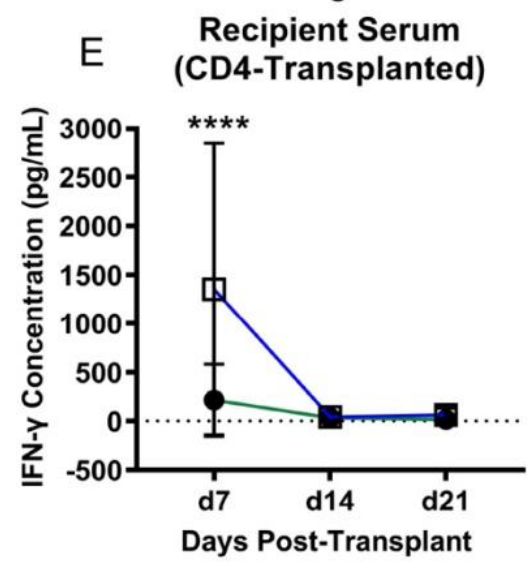

F Recipient Serum (CD4-Transplanted)

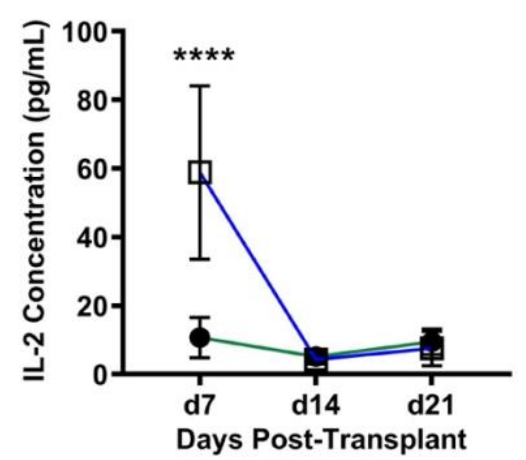

984

985 Figure 5: Loss of TCF-1 changes the cytokine profile of mature alloactivated CD4 T cells.

986 (A-B) Recipient Balb/c mice were allotransplanted with Balb/c BM and 1.5X106 WT or TCF

987 cKO donor CD3 T cells, as before. At day 7, splenocytes were obtained and restimulated by 6 
988 hours of culture with anti-CD3/anti-CD28 or PBS (control), along with GolgiPlug. Production of

989 (A) TNF- $\alpha$ and (B) IFN- $\gamma$ was measured by flow cytometry, using percent cytokine-positive

990 cells. Donor cells were stained for $\mathrm{H} 2 \mathrm{~Kb}, \mathrm{CD} 3, \mathrm{CD} 4$, and CD8, then fixed/permeabilized and

991 stained with anti-IFN- $\gamma$ and anti-TNF- $\alpha$. (C-F) Serum was obtained from cardiac blood of

992 euthanized recipient mice at day 7, day 14, and day 21 post-transplant, and was tested using a

993 LEGENDplex multiplex ELISA kit. (C) Serum concentration (pg/mL) of TNF- $\alpha$ over time for

994 WT versus TCF cKO-transplanted mice. (D) Serum concentration (pg/mL) of TNF- $\alpha$ at day 14

995 only for WT versus TCF cKO-transplanted mice. (E) Serum concentration $(\mathrm{pg} / \mathrm{mL})$ of IFN- $\gamma$

996 over time for WT versus TCF cKO-transplanted mice. (F) Serum concentration (pg/mL) of IL-2

997 over time for WT versus TCF cKO-transplanted mice. For A, B, and $\mathbf{D}$, individual points are

998 shown with mean and SD. For $\mathbf{C}, \mathbf{E}$, and $\mathbf{F}$, mean and SD only is shown. All data were analyzed

999 with one-way or two-way ANOVA or Student's t-test, and **** means p-value $\leq 0.0001 . \mathrm{N}=3-5$

1000 per group for A-B with data from two experiments shown, N=6 per group for C-F, summary data

1001 for C,E,F and individual points in D from one representative experiment. 

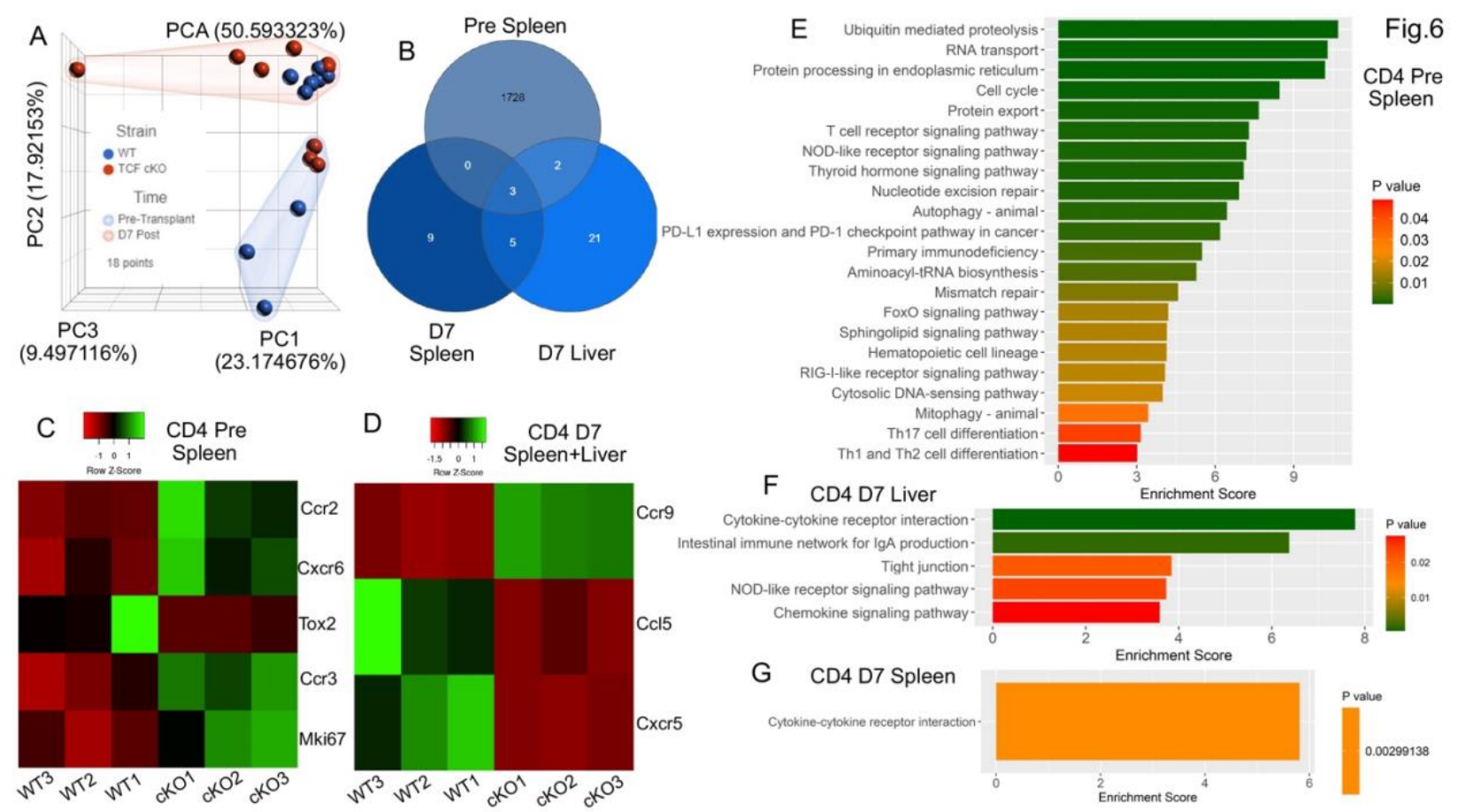

Figure 6: TCF-1 contributes to control of cytokine and immune signaling pathways in

1006

1007

1008

1009

1010

1011

1012

1013

1014

1015

1016

1017

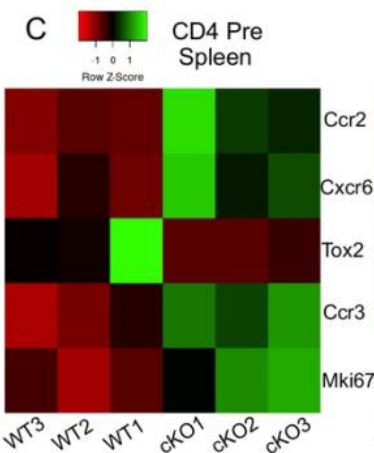

D $\quad \mathrm{CD} 4 \mathrm{D7}$

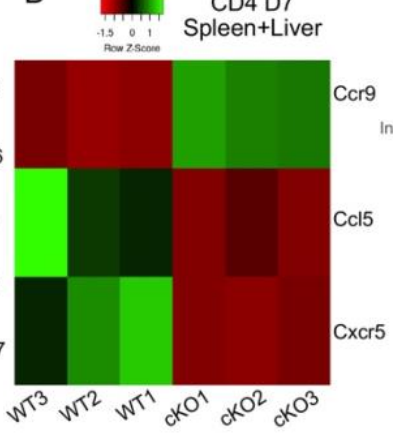

g.6

4 Pre

ve
0.04
0.03
0.02
0.01 
1018 altered pathways ( $\mathrm{p}$-value $\leq 0.05$ ) are shown, with enrichment score plotted and $\mathrm{p}$-value

1019 indicated by fill color. (E) Pre-transplant spleen, (F) Day 7 liver, and (G) Day 7 spleen are

1020 shown. $\mathrm{N}=3$ samples per group and condition, one experiment shown (done once).

1021 


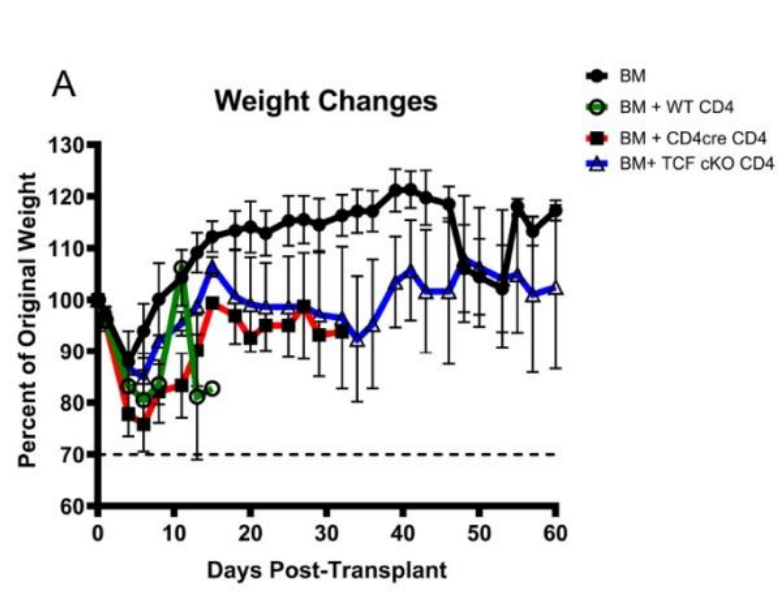

1023

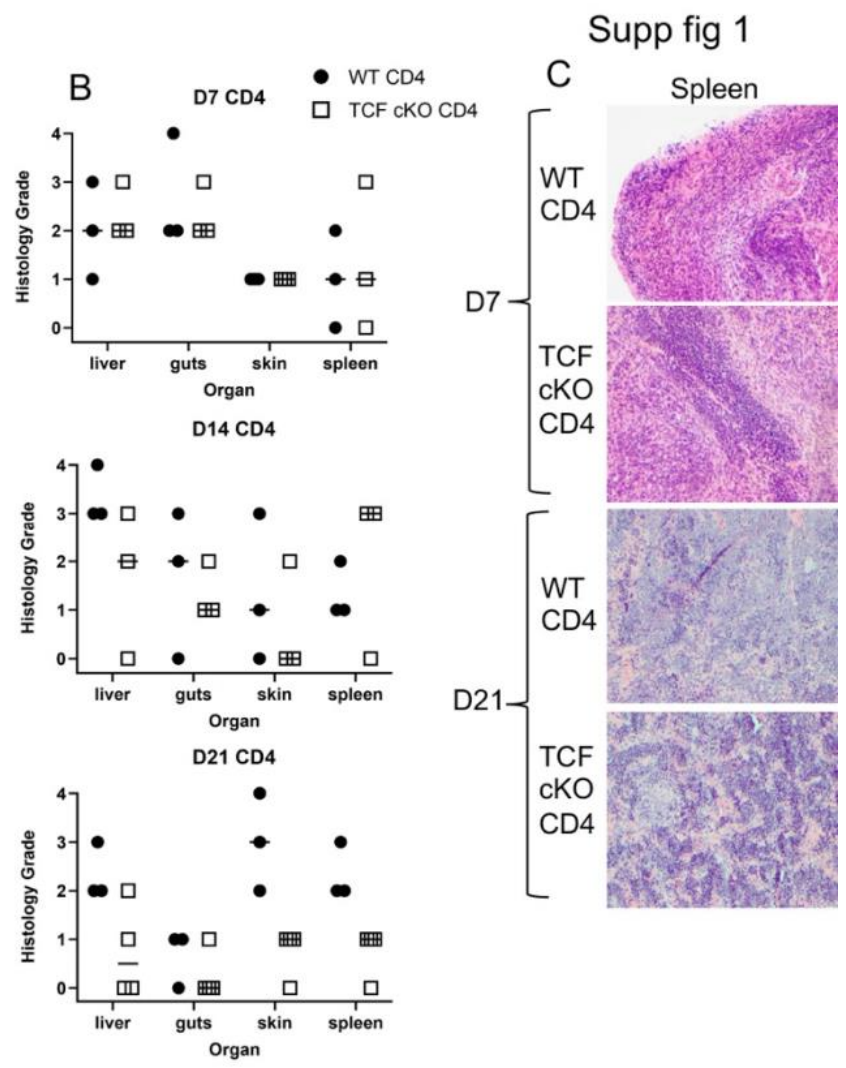

Supplemental Figure 1: GVHD symptoms and organ damage are reduced by loss of TCF-1,

related to Figure 1. Recipient mice were allotransplanted with BALB/c BM and WT, CD4cre,

or TCF cKO CD4 T cells as described in Fig. 1. (A) Weight loss for each group of mice over the section, with median indicated. (C) Spleen tissue sections from d7 and d21 are shown here. N=3representative photo shown in $\mathbf{C}$ and individual points in $\mathbf{B}$. 
A

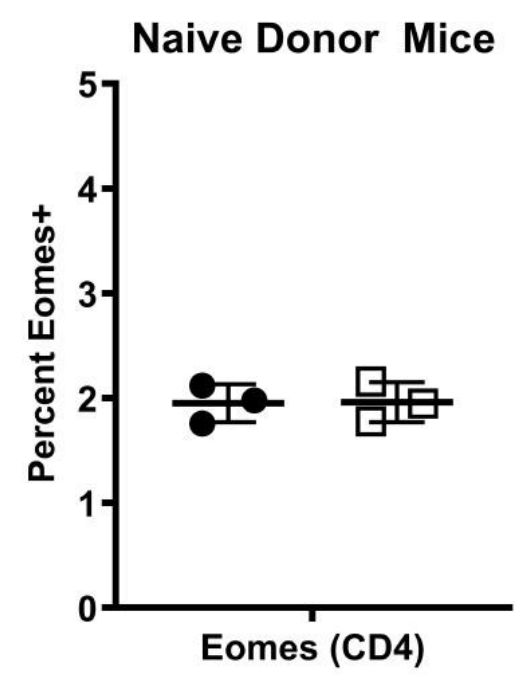

D Chimeric Mice

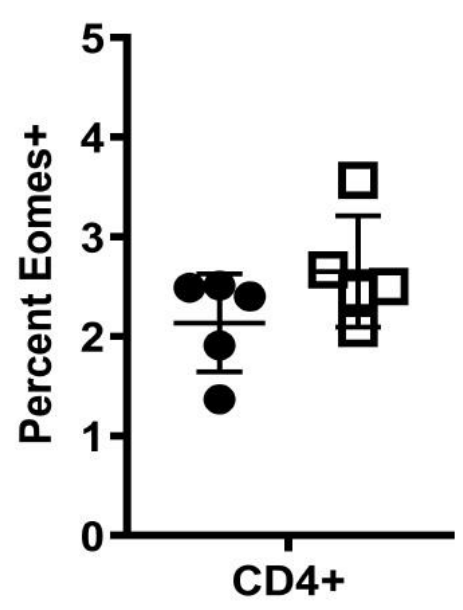

B

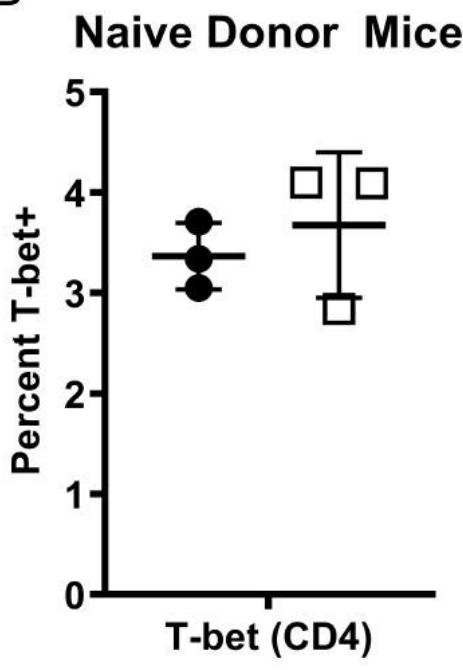

E Chimeric Mice

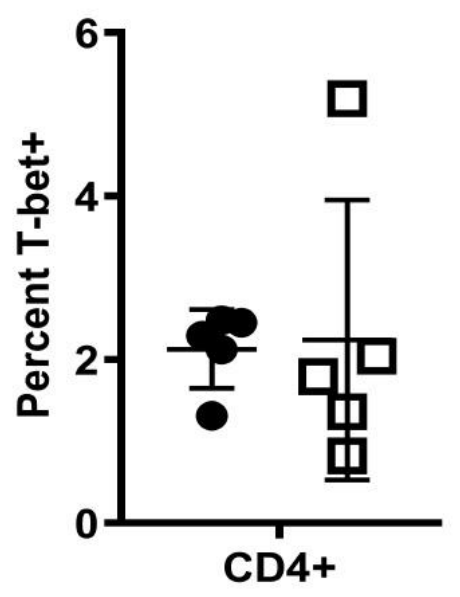

Supp fig 2

C

Naive Donor Mice

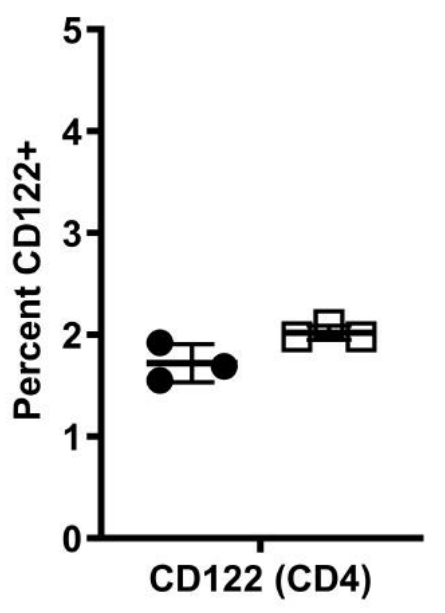

- WT

TCF KO

1034 Supplemental Figure 2: Loss of TCF-1 does not disrupt some phenotypic markers, related

1035 to Figure 2. (A-C) Splenocytes were taken from naive WT or TCF cKO donor mice and stained 1036 for flow cytometry. (A) Percent of CD4 T cells expressing Eomes. (B) Percent of CD4 T cells 1037 expressing T-bet. (C) Percent of CD4 T cells expressing CD122. (D-E) Thy1.1 mice were 1038 lethally irradiated and reconstituted with a 1:4 (WT:TCF cKO) mixture of bone marrow cells.

1039 Blood was checked by flow cytometry at 9 weeks to ensure reconstitution, and splenocytes were 1040 taken at 10 weeks for flow cytometry phenotyping. WT donor cells were identified by CD45.1, 
1041 while TCF cKO donor cells were identified by CD45.2 (D) Percent of chimeric CD4 T cells

1042 expressing Eomes. (E) Percent of chimeric CD4 T cells expressing T-bet. N=3-5 per group for

1043 A-C with one representative experiment shown, N=5 per group for D-E, one experiment shown 1044 (done once).

\section{Supp fig 3}

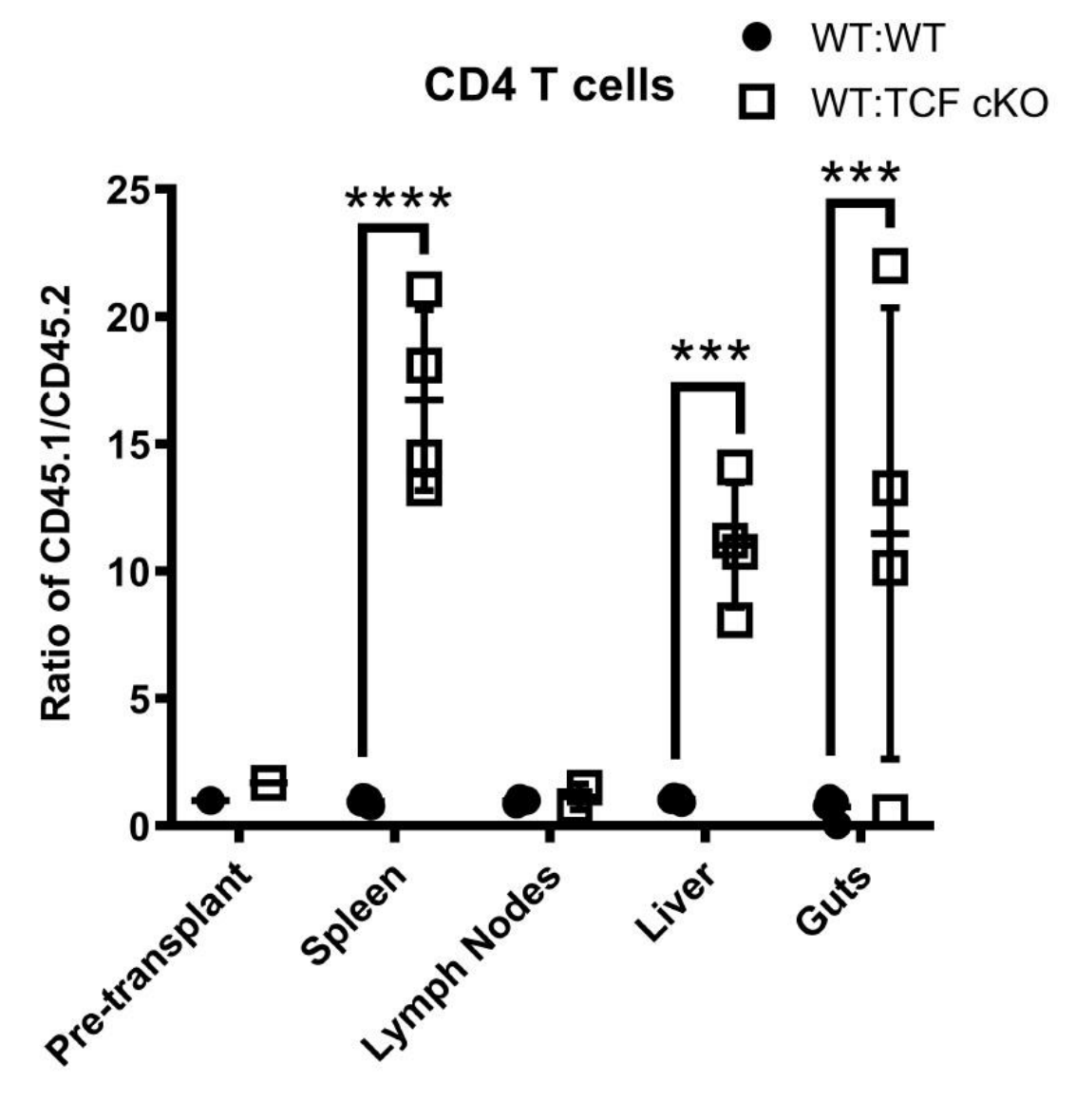

Supplemental Figure 3: Loss of TCF-1 alters migration of T cells, related to Figure 3.

1047 Recipient BALB/c mice were lethally irradiated and allotransplanted with $10 \mathrm{X} 10^{6}$ BALB/c bone

1048 marrow $(\mathrm{BM})$ cells and $1 \times 10^{6} \mathrm{CD} 3 \mathrm{~T}$ cells. The $\mathrm{T}$ cells were mixed at a 1:1 ratio of CD3 T cells

1049 from WT (CD45.1) and TCF cKO (CD45.2) donor mice, or WT (CD45.1) and WT (CD45.2) 

each).

\section{Supp fig 4}
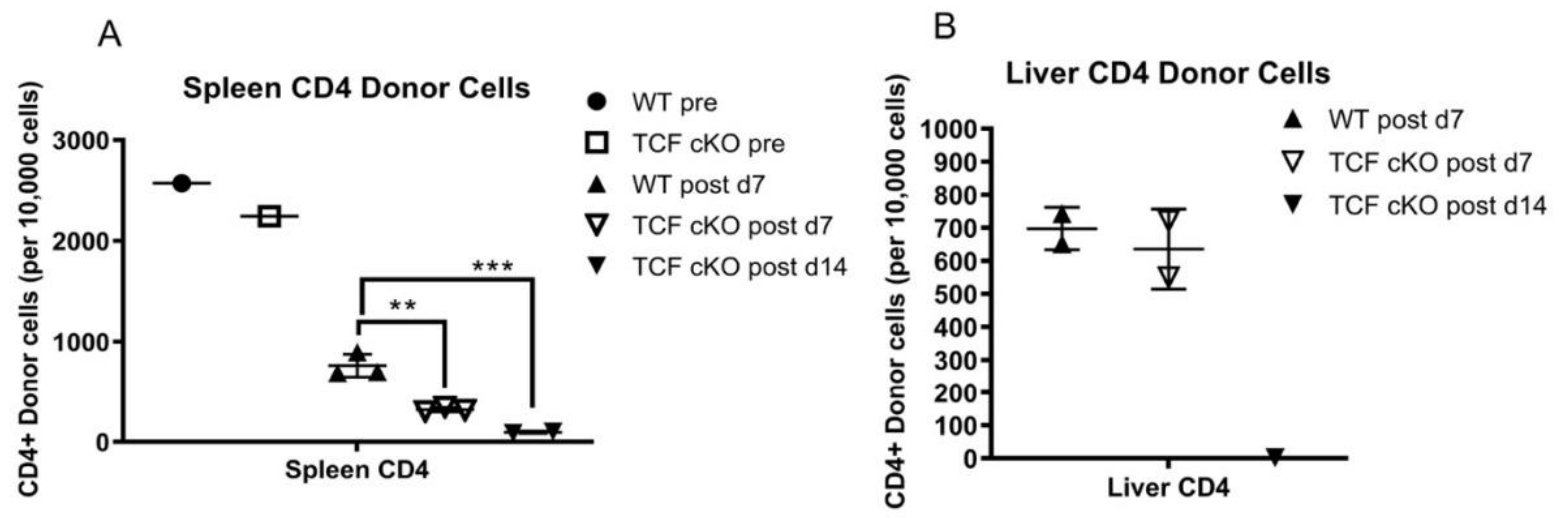

1057

Supplemental Figure 4: Donor cell numbers are reduced when TCF-1 is lost, related to

1059

1060

1061

1062

1063

1064

1065

Figure 4. Recipient mice were allotransplanted with BALB/c BM and WT or TCF cKO CD3 T cells. This was done for the RNA sequencing and qPCR experiments described, where donor cells must be FACS-sorted back from recipients post-transplant. Number of pre- and posttransplant CD4+ donor T cells obtained by sorting from donor or recipient mice (using antibodies for $\mathrm{H} 2 \mathrm{~Kb}, \mathrm{H} 2 \mathrm{Kd}, \mathrm{CD} 3, \mathrm{CD} 4$, and CD8) is shown per 10,000 sorted cells. Number of cells for each donor type and timepoint in (A) spleen and (B) liver, with individual points and mean with SD plotted. Data were analyzed with two-way ANOVA, ** means p-value $\leq 0.01$, 


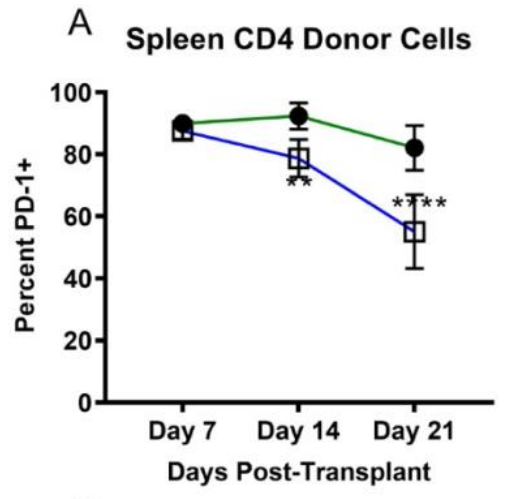

B

Liver CD4 Donor Cells

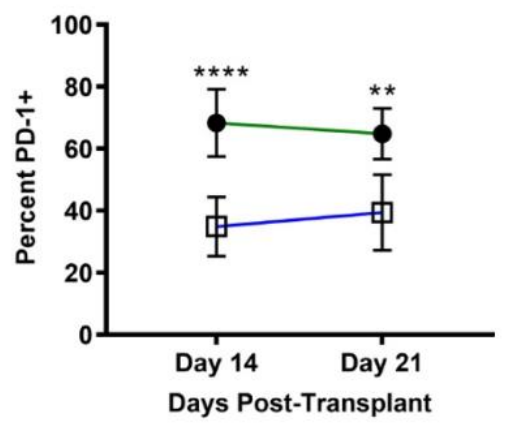

C Spleen CD4 Donor Cells

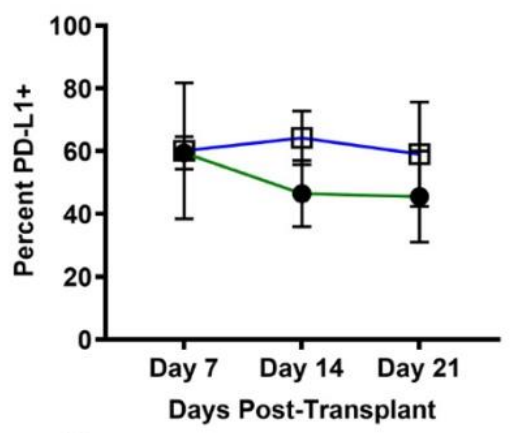

Liver CD4 Donor Cells

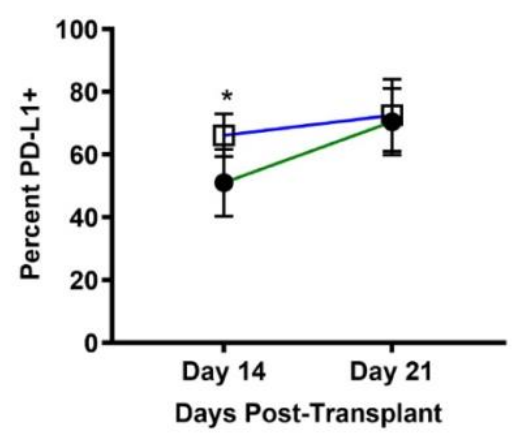

Supp fig 5

E Spleen CD4 Donor Cells

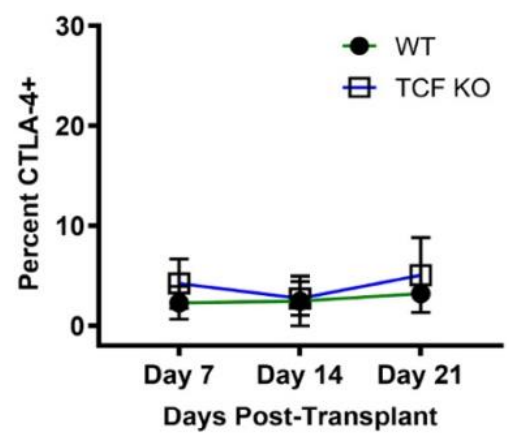

F Liver CD4 Donor Cells

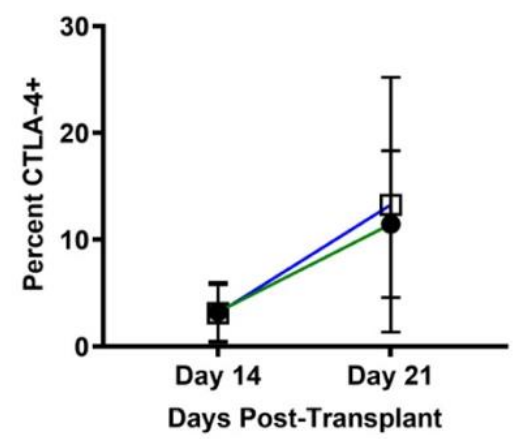

1069

Supplemental Figure 5: Loss of TCF-1 changes the suppressive profile of mature CD4 T

1070 cells, related to Figure 5. Recipient mice were allotransplanted with BALB/c BM and WT or

1071 TCF cKO CD4 donor T cells, as described before. At day 7, day 14, and day 21 post-transplant,

1072 spleens and livers (d14 and d21 only) were obtained from euthanized recipient mice.

1073 Lymphocytes were obtained and stained for $\mathrm{H} 2 \mathrm{~Kb}, \mathrm{CD} 3, \mathrm{CD} 4$, and CD8, as well as PD-1, PD-

1074 L1, and CTLA-4. (A) Expression of PD-1 on CD4+ donor cells in spleen over time. (B)

1075 Expression of PD-1 on CD4+ donor cells in liver over time. (C) Expression of PD-L1 on CD4+

1076 donor cells in spleen over time. (D) Expression of PD-L1 on CD4+ donor cells in liver over time.

1077 (E) Expression of CTLA-4 on CD4+ donor cells in spleen over time. (F) Expression of CTLA-4 

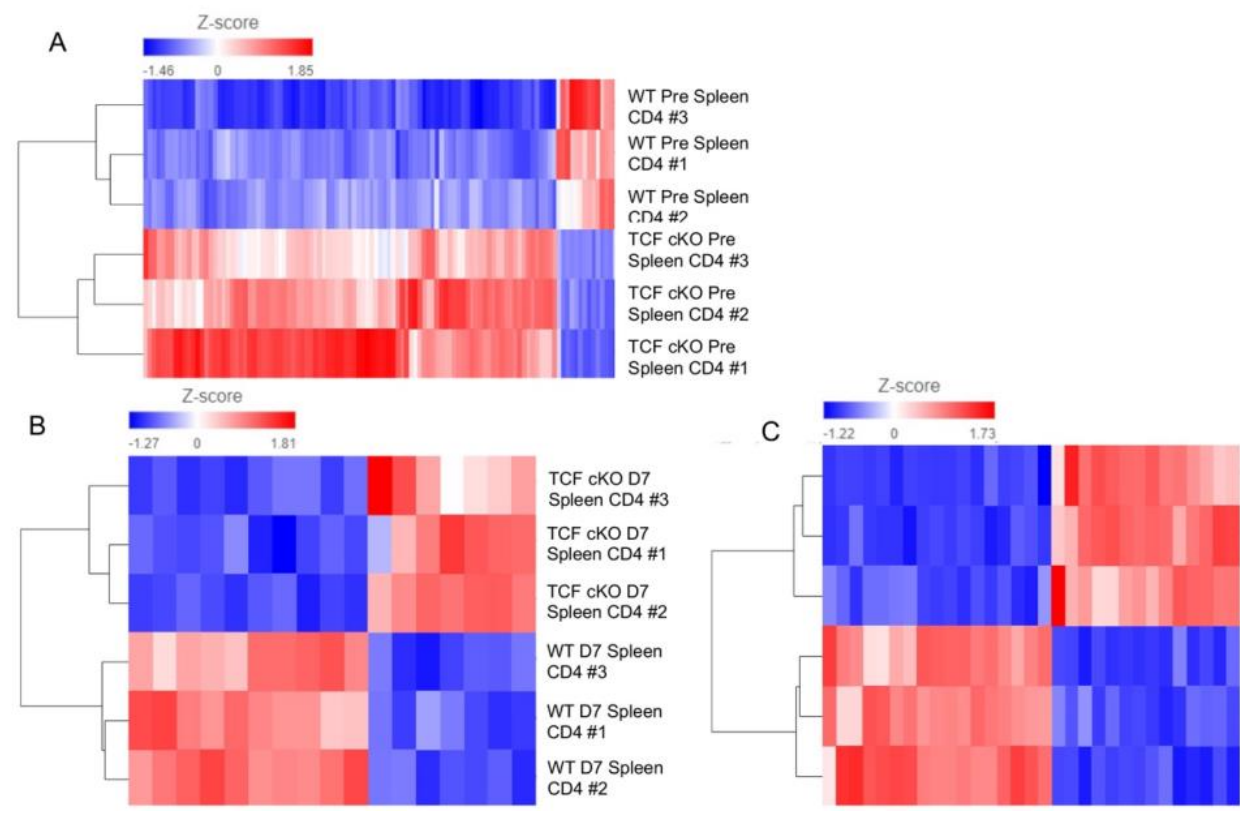

Supp Fig 6

TCF-1, related to Figure 6. As in Fig. 6, recipient BALB/c mice were allotransplanted with

7 post-transplant donor CD4 T cells were FACS-sorted from donor or recipient mice,

respectively. Cells were sorted into Trizol, RNA was extracted, paired-end sequencing was done, and data were analyzed in Partek Flow. Hierarchecal clustering was performed for (A) pretransplant spleen samples, (B) D7 spleen samples, and (C) D7 liver samples. Z-score scale used for heatmap is shown above each map. $\mathrm{N}=3$ per group and condition, one experiment shown

1091 (done once). 


\section{Wnt $\beta$-catenin pathway}

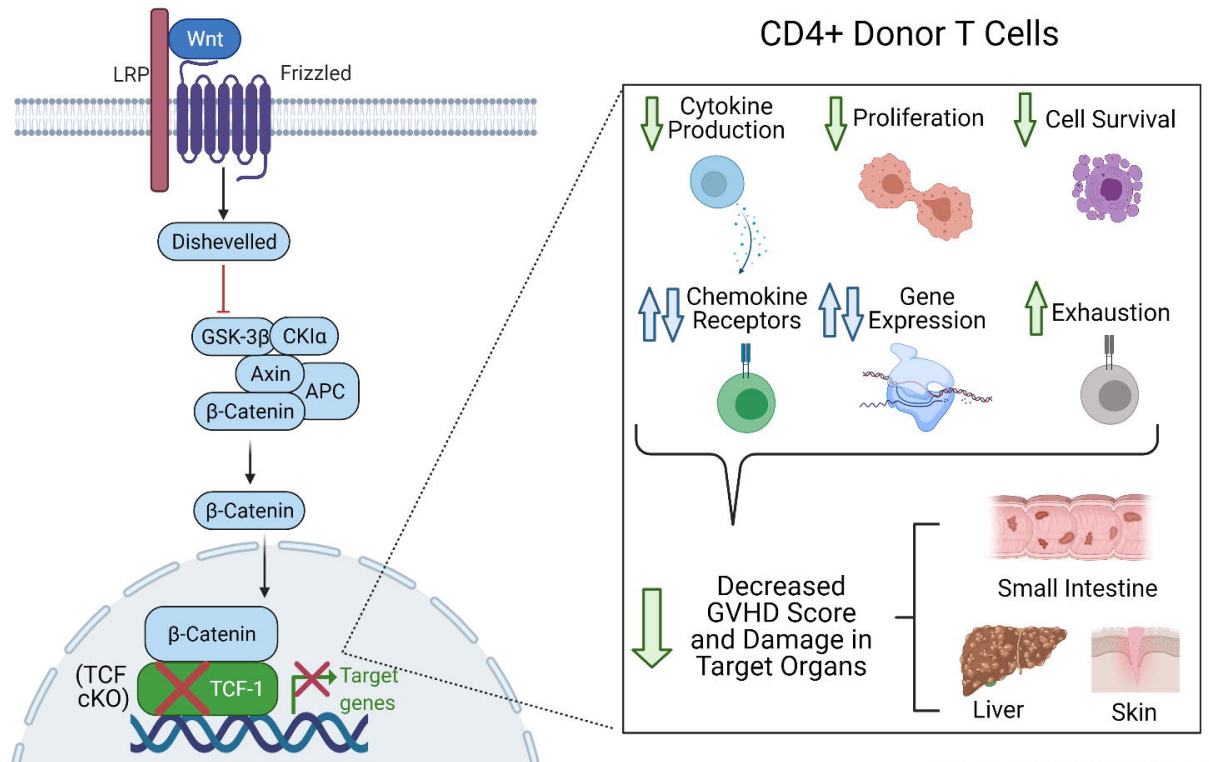

Created with Biorender.com 